\title{
ON INSTABILITY OF EXCITED STATES OF THE NONLINEAR SCHRÖDINGER EQUATION
}

\author{
SCIPIO CUCCAGNA
}

\begin{abstract}
We introduce a new notion of linear stability for standing waves of the nonlinear Schrödinger equation (NLS) which requires not only that the spectrum of the linearization be real, but also that the generalized kernel be not degenerate and that the signature of all the positive eigenvalues be positive. We prove that excited states of the NLS are not linearly stable in this more restrictive sense. We then give a partial proof that this more restrictive notion of linear stability is a necessary condition to have orbital stability.
\end{abstract}

\section{$\S 1$ INTRODUCTION}

We consider the NLS

$$
i u_{t}+\Delta u+\beta\left(|u|^{2}\right) u=0,(t, x) \in \mathbb{R} \times \mathbb{R}^{3}, u(0, x)=u_{0}(x) .
$$

We will assume the following hypotheses.

(H1) There exists an open interval $\mathcal{O} \subseteq(0,+\infty)$ such that $\Delta u-\omega u+\beta\left(u^{2}\right) u=0$ admits a family of standing waves $e^{i t \omega} \phi_{\omega}(x)$ with $\phi_{\omega}$ real valued and $\omega \in \mathcal{O} \rightarrow \phi_{\omega} \in$ $C^{1}\left(\mathcal{O}, H_{r}^{1}\left(\mathbb{R}^{3}\right)\right)$, with $H_{r}^{1}$ denoting radially symmetric finite energy functions.

(H2) $\beta(0)=0, \beta \in C^{1}(\mathbb{R}, \mathbb{R})$.

(H3) There exists a $1<p<5$ such that for every $k=0,1$,

$$
\left|\frac{d^{k}}{d v^{k}} \beta\left(v^{2}\right)\right| \lesssim|v|^{p-k-1} \quad \text { if }|v| \geq 1 .
$$

In this paper we focus on the question of orbital stability of the excited standing waves $e^{i t \omega} \phi_{\omega}$. This question has been explored thoroughly for ground states, i.e. when we can pick $\phi_{\omega}(x)>0$ for all $x$, see [CL,We1,GSS1-2] and countless other papers. In the case of ground states, orbital stability generally is equivalent to the 
standard notion of linear stability, which we review now. Recall that for $\sigma_{j}$ the Pauli matrices given below, then the linearization $H_{\omega}$ is given (see $\S 2$ ) by

$$
\begin{aligned}
& \sigma_{1}=\left[\begin{array}{ll}
0 & 1 \\
1 & 0
\end{array}\right], \sigma_{2}=\left[\begin{array}{cc}
0 & i \\
-i & 0
\end{array}\right], \sigma_{3}=\left[\begin{array}{cc}
1 & 0 \\
0 & -1
\end{array}\right], \\
& H_{\omega}=\sigma_{3}\left[-\Delta+\omega-\beta\left(\phi_{\omega}^{2}\right)-\beta^{\prime}\left(\phi_{\omega}^{2}\right) \phi_{\omega}^{2}\right]+i \beta^{\prime}\left(\phi_{\omega}^{2}\right) \phi_{\omega}^{2} \sigma_{2} .
\end{aligned}
$$

A ground state is generally orbitally stable if $\sigma\left(H_{\omega}\right) \subset \mathbb{R}$, but not always since there are well known cases like the critical equation $i u_{t}+\Delta u+|u|^{\frac{4}{n}} u=0$ with $\sigma\left(H_{\omega}\right) \subset \mathbb{R}$ where the generalized kernel of $H_{\omega}$ has higher degeneracy and the ground state is orbitally unstable. See also the work by Comech and Pelinovsky $[\mathrm{CoP}]$. In this paper we look at excited states. More precisely assume that the $\phi_{\omega}(x)$ are real valued and change sign. For these standing waves less appears to be known. One can look at the spectrum $\sigma\left(H_{\omega}\right)$ of the linearization (1.2), which is symmetric with respect of the coordinate axes. It has been known for a long time, but proved rigorously only recently by Mizumachi [M1] in dimension 2 (the argument extends to any dimension), that if $\sigma\left(H_{\omega}\right) \not \subset \mathbb{R}$ then $\phi_{\omega}$ is not orbitally stable in $H^{1}\left(\mathbb{R}^{n}\right)$. In the literature various studies of instability of standing waves are based on this form of linear instability, see [J,Gr1-2,M2-4]. Based on the above considerations, classically a standing wave is called linearly stable if $\sigma\left(H_{\omega}\right) \subset \mathbb{R}$. While this classical notion of linear stability is adequate in the case of ground states, our purpose here is to show that it is inadequate in the case of excited states, and to propose a substitute. In $\S 2$ Definition 2.3 we give a new definition of linear stability. Succinctly, this requires not only $\sigma\left(H_{\omega}\right) \subset \mathbb{R}$, but also that the generalized kernel $N_{g}\left(H_{\omega}\right)$ be not degenerate and that the signature of all positive eigenvalues be positive. This definition of linear stability generally coincides with the classical one in the case of ground states, because in that case $H_{\omega}$ has no positive eigenvalues of negative signature. However for excited states we have:

Theorem 1.1. Consider hypotheses (H1-3) and suppose that the $\phi_{\omega}$ are real valued and change sign. Then $\phi_{\omega}$ is not linearly stable in the sense of Definition 2.3.

The fact that excited states do not meet a new and more stringent definition of linear stability is by itself not very significant. What matters is to see whether this new definition sheds some light on the question of orbital instability of excited states. In this respect we conjecture that a standing wave $e^{i t \omega} \phi_{\omega}$ satisfying $(\mathrm{H} 1-3)$ and with $\phi_{\omega}$ real valued is orbitally stable if and only if it satisfies Definition 2.3 (we also conjecture that in that case it is also asymptotically stable in the sense of $[\mathrm{CM}])$. In sections 3 and 4 we establish in special situations part of the conjecture, that is that, under appropriate hypotheses, excited states with $\sigma\left(H_{\omega}\right) \subset \mathbb{R}$ are nonetheless orbitally unstable.

For an excited state with $\sigma\left(H_{\omega}\right) \subset \mathbb{R}$ there are three mechanisms which yield orbital instability, two known and a third one explored here. The first two mechanisms are basically linear, because in these two cases, even though $\sigma\left(H_{\omega}\right) \subset \mathbb{R}$, 
there are arbitrarily small perturbations of $H_{\omega}$, of appropriate restricted type, with eigenvalues outside $\mathbb{R}$. These first two mechanisms are also non generic. The third mechanism, the only one explored here, is nonlinear and applies to cases where the condition $\sigma\left(H_{\omega}\right) \subset \mathbb{R}$ is stable under perturbation. The first mechanism of instability arises from the degeneracy of the generalized kernel $N_{g}\left(H_{\omega}\right)$. This first mechanism is explored in $[\mathrm{CoP}]$ and is not discussed here. So in sections 3 and 4 we assume that $N_{g}\left(H_{\omega}\right)$ is non degenerate, which is a generic condition. The second mechanism of instability is related to the possible presence of eigenvalues of negative signature embedded in $\sigma_{c}\left(H_{\omega}\right)$. This phenomenon is absent for ground states. While we cannot point to examples in the literature of this occurrence for excited states, it should be possible to prove their existence via perturbation theory such as $[\mathrm{CHM}]$. Then orbital instability should follow by essentially linear mechanisms of the type in $[\mathrm{G} 1, \mathrm{TY} 4, \mathrm{CPV}]$. We do not discuss the above two mechanisms and we only say that if $N_{g}\left(H_{\omega}\right)$ is degenerate and there are embedded eigenvalues of negative signature, there are additional reasons for instability with respect to the ones described here. Furthermore, if present, the first two mechanisms will usually prevail, because usually they unfold more rapidly than the third. The third mechanism is nonlinear and robust. The setting is related to attempts in a long list of papers [BP,SW2,TY1-3,Cu3,BS,T,GS,SW1,CM,Cu1,CT] to prove asymptotic stability of stable ground states. We assume more regularity on the nonlinearity $\beta(r)$. This because we consider appropriate Taylor expansions of $\beta\left(|w|^{2}\right) w$ and normal forms transformations which lead to a particular expansion of equation (1.1) around the orbit of an excited state. There is a natural decomposition in discrete and continuous modes, with the discrete ones satisfying a perturbation of a Hamiltonian system. In sections 3 and 4 it is described, after [BP,SW2,TY1-3,Cu3,BS,T,SW1,GS,CM], a possible mechanism through which the coupling of discrete with the continuous modes breaks the conservation laws of the unperturbed system of discrete modes and yields, in sections 3 and 4, orbital instability of excited states. This mechanism is called Nonlinear Fermi Golden Rule (FGR), after Sigal [Si]. In section 3 we consider the case when the portion $\sigma_{d}\left(H_{\omega}\right) \backslash\{0\}$ of the discrete spectrum is close to the continuous spectrum $\sigma_{c}\left(H_{\omega}\right)$. In this case our proof is valid generically. In section $4, \sigma_{d}\left(H_{\omega}\right) \backslash\{0\}$ is not any more close to $\sigma_{c}\left(H_{\omega}\right)$ and our proof hinges on a conjecture on the FGR, which we assume as hypothesis in Hypothesis 4.4 and is related to similar conjectures in $[\mathrm{GS}, \mathrm{Gz}, \mathrm{CM}]$. Notice that even though the conjecture on the FGR in our present setting gives orbital instability, in other settings, see the papers just referenced, this same mechanism yields asymptotic stability. The FGR can be viewed as a consequence of identities between some coefficients in the system on continuous and discrete modes. These are Taylor coefficients of the right hand sides of the equations. If the system derived from a real valued Hamiltonian $\mathcal{H}$, these coefficients would be mixed derivatives of $\mathcal{H}$, with different order of differentiation, and would be equal by the Schwarz lemma on mixed derivatives. Notice also that the NLS (1.1) is derived from a real valued Hamiltonian. Unfortunately 
we are not able to retain this Hamiltonian structure during the normal forms argument. Therefore the FGR remains a conjecture. Another ingredient required is that the above mentioned coefficients do not vanish on appropriate spheres of the phase space associated to $H_{\omega}$, see for instance (3.6). In this respect, we refer to a question in p.69 [SW2] on the relation between vanishing and integrability. At least in the non integrable setting we hope to have identified the mechanisms of instability. The same proof up to cosmetic changes can be used for non translation invariant equations of the form

$$
i u_{t}+(\Delta-q(x)) u+a(x) \beta\left(|u|^{2}\right) u=0
$$

for $q(x)$ short range and regular and $a(x)$ regular and bounded. When $-\Delta+q(x)$ has two or more negative eigenvalues, it is easy to manufacture by bifurcation, in the spirit of [SW1-2,TY1-4,T] for systems of the form (1.3), small excited states for which our proof of orbital instability holds. But our proof is valid more generally.

In the sequel a matrix will be called real when its components are real valued. A matrix will be exponentially decreasing when its components are functions converging exponentially to 0 as $|x| \rightarrow \infty$. For $f(x)$ and $g(x)$ column vectors, their inner product is $\langle f, g\rangle=\int_{\mathbb{R}^{d}}{ }^{t} f(x) \cdot \bar{g}(x) d x$ with $\bar{g}$ the vector with entries which are complex conjugate and with ${ }^{t} f$ the transposed vector. The adjoint $H^{*}$ is defined by $\langle H f, g\rangle=\left\langle f, H^{*} g\right\rangle$. By $L^{p}\left(\mathbb{R}^{3}\right)$ or $L^{p}$ we will denote not only the usual $L^{p}\left(\mathbb{R}^{3}, \mathbb{C}\right)$ but also $L^{p}\left(\mathbb{R}^{3}, \mathbb{C}^{2}\right)$, with the exact meaning clarified by the context. Same convention for the Sobolev spaces $W^{k, p}$, with $H^{k}=W^{k, 2}$. For $\langle x\rangle=\sqrt{1+|x|^{2}}$ we will consider weighted norms $\|u\|_{L^{2, s}}=\left\|\langle x\rangle^{s} u\right\|_{L^{2}\left(\mathbb{R}^{3}\right)}$, mixed norms $\|g(t, x)\|_{L_{t}^{p} L_{x}^{q}}=$ \|\|$g(t, x)\left\|_{L_{x}^{q}}\right\|_{L_{t}^{p}}$ and $\|g(t, x)\|_{L_{t}^{p} L_{x}^{2, s}}=\|\| g(t, x)\left\|_{L_{x}^{2, s}}\right\|_{L_{t}^{p}}$. Given a norm $\|g\|_{L_{t}^{p} L_{x}^{q}}$ the

pair $(p, q)$ is said to be admissible if $\frac{1}{p}=\frac{3}{2}\left(\frac{1}{2}-\frac{1}{q}\right)$ with $2 \leq q \leq 6$. Given an operator $H$, we set $R_{H}(z)=(H-z)^{-1}$. In the sequel, for $\lambda \in \mathbb{R}$ we will write $R_{H}^{ \pm}(\lambda)=R_{H}(\lambda \pm i 0)$ with on the left an appropriate (i.e. radial or nontangential) limit of $R_{H}(z)$ for $z \rightarrow \lambda$ with $\pm \Im z>0$. Here given a complex number $z=x+i y$, we set $\Re z=x$ and $\Im z=y$. For a matrix or vector $A$, we denote by ${ }^{t} A$ the transpose.

\section{$\S 2$ Definition of Linear Stability And Proof of Theorem 1.1}

We can write the following ansatz:

$$
u(t, x)=e^{i \vartheta(t)}\left(\phi_{\omega(t)}(x)+r(t, x)\right) \text { with } \vartheta(t)=\int_{0}^{t} \omega(s) d s+\gamma(t) .
$$

Inserting (2.1) in (1.1) we get for some $n(r, \bar{r})=O\left(r^{2}\right), \overline{n(r, \bar{r})}=n(\bar{r}, r)$

$$
\begin{aligned}
& i r_{t}=-\Delta r+\omega(t) r-\beta\left(\phi_{\omega(t)}^{2}\right) r-\beta^{\prime}\left(\phi_{\omega(t)}^{2}\right) \phi_{\omega(t)}^{2} r \\
& -\beta^{\prime}\left(\phi_{\omega(t)}^{2}\right) \phi_{\omega(t)}^{2} \bar{r}+\dot{\gamma}(t) \phi_{\omega(t)}-i \dot{\omega}(t) \partial_{\omega} \phi_{\omega(t)}+\dot{\gamma}(t) r+n(r, \bar{r}) .
\end{aligned}
$$


Set ${ }^{t} R=(r, \bar{r}),{ }^{t} \Phi_{\omega}=\left(\phi_{\omega}, \phi_{\omega}\right),{ }^{t} N(R)=(n(r, \bar{r}),-\bar{n}(r, \bar{r}))$. We rewrite the equation for $r$ as

$$
\begin{aligned}
i R_{t} & =H_{\omega(t)} R+\sigma_{3} \dot{\gamma}(t) R+\sigma_{3} \dot{\gamma}(t) \Phi_{\omega}-i \dot{\omega}(t) \partial_{\omega} \Phi_{\omega}+N(R) . \\
H_{\omega} & =\sigma_{3}\left[-\Delta+\omega-\beta\left(\phi_{\omega}^{2}\right)-\beta^{\prime}\left(\phi_{\omega}^{2}\right) \phi_{\omega}^{2}\right]+i \sigma_{2} \beta^{\prime}\left(\phi_{\omega}^{2}\right) \phi_{\omega}^{2} .
\end{aligned}
$$

For an operator $L$ the generalized kernel is the space $N_{g}(L)=\cup_{j \geq 1} \operatorname{ker}\left(L^{j}\right)$. We have:

(a) $\sigma_{1} H_{\omega}=-H_{\omega} \sigma_{1}$

(b) if we set $H_{\omega}=\sigma_{3}(-\Delta+\omega)+V_{\omega}(x)$, then the matrix $V_{\omega}(x)$ has real valued entries.

(a)-(b) imply that point spectrum $\sigma_{p}\left(H_{\omega}\right)$ and essential spectrum $\sigma_{e}\left(H_{\omega}\right)$ are symmetric with respect to the coordinate axes. The following standard lemma, which requires the exponential decay of $\phi(x)$ at infinity, is proved in [CPV]:

Lemma 2.1. The point spectrum is a finite set and we have $\sigma_{p}\left(H_{\omega}\right)=\sigma_{p}\left(H_{\omega}^{*}\right)$. Similarly for the essential spectrum $\sigma_{e}\left(H_{\omega}\right)=\sigma_{e}\left(H_{\omega}^{*}\right)=(-\infty,-\omega] \cup[\omega,+\infty)$. For each $z \in \sigma_{p}\left(H_{\omega}\right)$ the corresponding generalized eigenspace $N_{g}\left(H_{\omega}-z\right)$ has finite dimension.

We are assuming that $\sigma\left(H_{\omega}\right) \subset \mathbb{R}$ because otherwise by [M1] the standing wave is unstable. We define:

Definition 2.2. Let $\lambda>0$ be an eigenvalue of $H_{\omega}$. We say that $\lambda$ has positive (resp. negative) signature if the following two points hold:

(1) the algebraic and geometric multiplicity coincide, i.e. $N_{g}\left(H_{\omega}-\lambda\right)=\operatorname{ker}\left(H_{\omega}-\lambda\right)$;

(2) for any $\xi \in \operatorname{ker}\left(H_{\omega}-\lambda\right)$ with $\xi \neq 0$ we have $\left\langle\xi, \sigma_{3} \xi\right\rangle>0$ (resp. $\left.\left\langle\xi, \sigma_{3} \xi\right\rangle<0\right)$.

Remark. Notice that if $z \in \sigma_{p}\left(H_{\omega}\right) \backslash \mathbb{R}$ then $\left\langle\xi, \sigma_{3} \xi\right\rangle=0$ for any $\xi \in \operatorname{ker}\left(H_{\omega}-z\right)$.

Remark. We are unable to reference examples of eigenvalues $\lambda>\omega$ with negative signature so we sketch what seems a natural way to manufacture them. Consider a short range Schrödinger operator $h=-\Delta+q(x)$ with $\sigma_{d}(h) \supseteq\left\{-E_{0},-E_{1}\right\}$, with $-E_{0}$ the smallest eigenvalue and with $-E_{0}<-E_{1}<0$. By bifurcation, equation (1.3) with $a(x) \equiv 1$ will have small amplitude excited states $e^{i \omega t} \phi_{\omega}(x)$ where $\omega \in \Omega, \Omega$ a small open interval with $E_{1}$ an endpoint. $\sigma_{3}(h+\omega)$ will have a real negative signature eigenvalue $E_{0}-\omega$. This will be in $[\omega, \infty)$ if $E_{0}-E_{1}>E_{1}$. We have that $H_{\omega}=\sigma_{3}(h+\omega)+V\left(\phi_{\omega}\right)$ with $V\left(\phi_{\omega}\right)(x)=\sigma_{3} \varphi_{\omega}(x)+i \sigma_{2} \psi_{\omega}(x)$ with $\phi_{\omega}$ and $\psi_{\omega}$ real valued exponentially decreasing functions. Generically, by $[\mathrm{G} 1, \mathrm{TY} 4, \mathrm{CPV}], H_{\omega}$ will have a pair of non real eigenvalues close to $E_{0}-\omega$. However, in analogy to the conjecture in the context of the $N$ body problem in [AHS], there is a hypersurface $\Sigma$ of pairs $\left(\phi_{\omega}, \psi_{\omega}\right)$ such that $H_{\omega}=\sigma_{3}(h+\omega)+\sigma_{3} \varphi_{\omega}(x)+i \sigma_{2} \psi_{\omega}(x)$ has a real eigenvalue $\lambda(\omega)$ near $E_{0}-\omega$, of negative signature. This is easy to see by employing the Weinstein-Aroszajn formula in $\S 5[\mathrm{CPV}]$ which relates $\lambda(\omega)$ to $\left(\phi_{\omega}, \psi_{\omega}\right)$. Furthermore it should be possible to find some $\beta\left(|u|^{2}\right) u$ in (1.3) such 
that we have $V\left(\phi_{\omega}\right)(x)=\sigma_{3} \varphi_{\omega}(x)+i \sigma_{2} \psi_{\omega}(x)$ with $\left(\phi_{\omega}, \psi_{\omega}\right) \in \Sigma$. Notice that the conjecture in [AHS], proved for Wigner-Von Neuman potentials in [CHM], is in a setting much harder then ours since we are considering only very short range potentials.

Remark. It is not known if there are eigenvalues $\lambda>\omega$ with positive signature. We conjecture that they do not exist.

Remark. In [CPV] it is proved that generically eigenvalues $\lambda>\omega$ do not exist in our setting, that is with matrix potentials $V_{\omega}(x)=\sigma_{3} \varphi_{\omega}(x)+i \sigma_{2} \psi_{\omega}(x)$ with $\phi_{\omega}$ and $\psi_{\omega}$ real valued exponentially decreasing functions.

We introduce now our definition of linear stability. The usual definition is that $\sigma\left(H_{\omega}\right) \subset \mathbb{R}$. We prefer the following more stringent definition.

Definition 2.3(Linear stability). We will say that $\phi_{\omega}$ is linearly stable if the operator $H_{\omega}$ satisfies the following three conditions:

(1) $\sigma\left(H_{\omega}\right) \subset \mathbb{R}$;

(2) if $\lambda>0$ is an eigenvalue of $H_{\omega}$ then $\lambda$ has positive signature;

(3) $N_{g}\left(H_{\omega}\right)$ is spanned by $\left\{\sigma_{3} \Phi_{\omega}, \partial_{\omega} \Phi_{\omega}, \partial_{x} \Phi_{\omega}, \sigma_{3} x \Phi_{\omega}\right\}$.

Remark. That $N_{g}\left(H_{\omega}\right) \supseteq \operatorname{span}\left\{\sigma_{3} \Phi_{\omega}, \partial_{\omega} \Phi_{\omega}, \partial_{x} \Phi_{\omega}, \sigma_{3} x \Phi_{\omega}\right\}$ follows by direct computation. The fact that generically $N_{g}\left(H_{\omega}\right)$ strictly larger than this span implies orbital instability has been explored in $[\mathrm{CoP}]$. In sections 3 and 4 we will assume that, in the context of even functions, $N_{g}\left(H_{\omega}\right)=\operatorname{span}\left\{\sigma_{3} \Phi_{\omega}, \partial_{\omega} \Phi_{\omega}\right\}$.

Remark. $N_{g}\left(H_{\omega}\right)=\operatorname{span}\left\{\sigma_{3} \Phi_{\omega}, \partial_{\omega} \Phi_{\omega}, \partial_{x} \Phi_{\omega}, \sigma_{3} x \Phi_{\omega}\right\}$ is proved in [We2] under hypothesis (H4), see $\S 3$, and if $\frac{d}{d \omega}\left\|\phi_{\omega}\right\|_{2} \neq 0$.

Remark. If we break the translation invariance of the equation, then (3) is replaced by $N_{g}\left(H_{\omega}\right)=\operatorname{span}\left\{\sigma_{3} \Phi_{\omega}, \partial_{\omega} \Phi_{\omega}\right\}$.

Proof of Theorem 1.1. Set $L_{+}=-\Delta+\omega-\beta\left(\phi_{\omega}^{2}\right)-2 \phi_{\omega}^{2} \beta^{\prime}\left(\phi_{\omega}^{2}\right)$ and $L_{-}=$ $-\Delta+\omega-\beta\left(\phi_{\omega}^{2}\right)$. For $U=\left[\begin{array}{cc}1 & 1 \\ i & -i\end{array}\right]$ we have $U \sigma_{3} H_{\omega} U^{-1}=\operatorname{diag}\left(L_{+}, L_{-}\right)$. Notice that $L_{-} \phi_{\omega}=0$. Since $\phi_{\omega}$ has nodes, $L_{-}$has a smallest strictly negative eigenvalue. The corresponding ground states of $L_{-}$are spherically symmetric. $L_{+} \partial_{x_{j}} \phi_{\omega}=0$ for all $j$ and so also $L_{+}$has a smallest strictly negative eigenvalue with corresponding ground states which are spherically symmetric. From now on in this proof we consider $H_{\omega}, L_{+}$and $L_{-}$as acting on spherically symmetric functions only. Let

$$
N\left(\sigma_{3} H_{\omega}\right)=\sum_{\lambda \in \sigma_{p}\left(\sigma_{3} H_{\omega}\right) \cap(-\infty, 0)} \operatorname{dim} \operatorname{ker}\left(\sigma_{3} H_{\omega}-\lambda\right) .
$$


Since we are restricting to spherically symmetric functions, condition (3) becomes $N_{g}\left(H_{\omega}\right)=\operatorname{span}\left\{\sigma_{3} \Phi_{\omega}, \partial_{\omega} \Phi_{\omega}\right\}$. The fact, discussed above, that for both signs we have $\sigma\left(L_{ \pm}\right) \cap(-\infty, 0) \neq \emptyset$, implies $N\left(\sigma_{3} H_{\omega}\right) \geq 2$. We claim now that if $H_{\omega}$ satisfies the conditions in Definition 2.3 we have

$$
\left\langle\sigma_{3} H_{\omega} u, u\right\rangle \geq 0 \text { for any non zero } u \in H^{1} \cap N_{g}^{\perp}\left(H_{\omega}^{*}\right)
$$

Before proving (2.3), we show that (2.3) implies $N\left(\sigma_{3} H_{\omega}\right) \leq 1$. We have an $H_{\omega}$ invariant splitting $L^{2}=N_{g}\left(H_{\omega}\right) \oplus N_{g}^{\perp}\left(H_{\omega}^{*}\right)$ with $\sigma_{3} N_{g}^{\perp}\left(H_{\omega}^{*}\right)=N_{g}^{\perp}\left(H_{\omega}\right)$. This implies that given a generic $u=v+w \in N_{g}\left(H_{\omega}\right) \oplus N_{g}^{\perp}\left(H_{\omega}^{*}\right)$,

$$
\left\langle\sigma_{3} H_{\omega} u, u\right\rangle=\left\langle\sigma_{3} H_{\omega} v, v\right\rangle+\left\langle\sigma_{3} H_{\omega} w, w\right\rangle \geq\left\langle\sigma_{3} H_{\omega} v, v\right\rangle
$$

By (3) Definition 2.3 we have $v=\lambda \sigma_{3} \Phi+\mu \partial_{\omega} \Phi$ with

$$
\begin{aligned}
& \left\langle\sigma_{3} H_{\omega} v, v\right\rangle=\lambda^{2}\left\langle\sigma_{3} H_{\omega} \sigma_{3} \Phi, \sigma_{3} \Phi\right\rangle+\lambda \mu\left[\left\langle\sigma_{3} H_{\omega} \sigma_{3} \Phi, \partial_{\omega} \Phi\right\rangle+\left\langle\sigma_{3} H_{\omega} \partial_{\omega} \Phi, \sigma_{3} \Phi\right\rangle\right] \\
& +\mu^{2}\left\langle\sigma_{3} H_{\omega} \partial_{\omega} \Phi, \partial_{\omega} \Phi\right\rangle=-\mu^{2} \partial_{\omega}\|\phi\|_{2}^{2}
\end{aligned}
$$

by $H_{\omega} \partial_{\omega} \Phi=-\sigma_{3} \Phi$. So $N\left(\sigma_{3} H_{\omega}\right) \leq 1$, which is incompatible with $N\left(\sigma_{3} H_{\omega}\right) \geq 2$. To conclude the proof of Theorem 1.1 we need to prove (2.3):

Proposition 2.4. If $H_{\omega}$ satisfies the conditions in Definition 2.3 for any non zero $u \in H^{1} \cap N_{g}^{\perp}\left(H_{\omega}^{*}\right)$ we have $\left\langle\sigma_{3} H_{\omega} u, u\right\rangle \geq 0$.

Under our hypotheses we have the decomposition

$$
\begin{aligned}
& N_{g}^{\perp}\left(H_{\omega}^{*}\right)=\sum_{\lambda \in \sigma_{p} \backslash\{0\}} \operatorname{ker}\left(H_{\omega}-\lambda\right) \oplus L_{c}^{2}\left(H_{\omega}\right) \\
& \text { where } L_{c}^{2}\left(H_{\omega}\right)=\left\{N_{g}\left(H_{\omega}^{*}\right) \oplus \sum_{\lambda \in \sigma_{p} \backslash\{0\}} \operatorname{ker}\left(H_{\omega}^{*}-\lambda\right)\right\}^{\perp} .
\end{aligned}
$$

We have $\left\langle\sigma_{3} H_{\omega} u, v\right\rangle=0$ for $u$ and $v$ in different terms in (1). By hypothesis, $\left\langle\sigma_{3} H_{\omega} \cdot, \cdot\right\rangle$ is a positive quadratic form in each $\operatorname{ker}\left(H_{\omega}-\lambda\right)$. So Proposition 2.4 is a consequence of

$$
\left\langle\sigma_{3} H_{\omega} u, u\right\rangle \geq 0 \text { for any } u \in H^{1} \cap L_{c}^{2}\left(H_{\omega}\right)
$$

(2.4) is a general fact. In this section we will consider some special cases for $H_{\omega}$ and in $\S 5$ we will complete the proof of (2.4). First of all we remind the following definition: 
Definition 2.5. $\omega$ is a resonance if there is a distribution $F$ such that $H_{\omega} F=\omega F$ such that $F \in L^{2,-s}$ for any $s>1 / 2$ but $F \notin L^{2}$.

If $\omega$ is neither a resonance nor an eigenvalue of $H_{\omega}$, which is a generic condition, then by Theorem $2.11[\mathrm{CPV}]$ in (2.4) we have $\left\langle\sigma_{3} H_{\omega} u, u\right\rangle>0$ for $u \neq 0$. We consider now the case when $\omega$ is a resonance or an eigenvalue. Using the terminology in Jensen and Kato [JK] we can distinguish between $\omega$ being exceptional point of first kind (when $\omega$ is a resonance but not an eigenvalue), second kind (when $\omega$ is an eigenvalue but not a resonance) and third kind ( $\omega$ both resonance and eigenvalue). In this section we consider two special cases. The proof is then completed in $\S 5$.

Lemma 2.6. Let $f, g \in C_{0}^{\infty}\left(\mathbb{R}^{3}, \mathbb{R}\right)$ and set $U_{1}(x)=\sigma_{3} f+i g \sigma_{2}$. Suppose that in the space $\mathcal{V}$ formed by eigenfunctions and resonant functions at $\omega$ the quadratic form

$$
\left\langle\sigma_{3} U_{1} \cdot, \cdot\right\rangle \text { is strictly positive. }
$$

Suppose furthermore that $\omega$ is either exceptional of first kind or of second type. If $\omega$ is of second type assume furthermore that $\operatorname{dim} \mathcal{V}=1$. Then for any $\varepsilon>0$ sufficiently close to 0 we have:

(1) the point $\omega$ is neither a resonance nor an eigenvalue for $H_{\omega, \varepsilon}:=H_{\omega}+\varepsilon U_{1}$.

(2) $H_{\omega, \varepsilon}$ does not have eigenvalues close to $\omega$.

Lemma 2.6 is proved $[\mathrm{CuP}]$. Notice that $\operatorname{dim} \mathcal{V}<\infty$ and probably the statement holds always without the restriction $\operatorname{dim} \mathcal{V}=1$. Nonetheless, in $\S 5$ we give a different proof of the remaining cases (2.4). Assuming the conclusions of Lemma 2.6, which are valid for $\operatorname{dim} \mathcal{V}=1$, by $\operatorname{dim} \mathcal{V}<\infty$ whe know that there are $U_{1}$ as above satisfying (2.5). Let now $\gamma$ be a fixed and small counterclockwise circle with center the origin in $\mathbb{C}$.

$$
\text { Set } \begin{aligned}
& P_{\varepsilon}:=-\frac{1}{2 \pi i} \int_{\gamma} R_{H_{\omega, \varepsilon}}(z) d z=P^{(0)}-\varepsilon P^{(1)}+\varepsilon^{2} P(\varepsilon) \\
& \\
& P^{(0)} \text { is the projection on } N_{g}\left(H_{\omega}\right) \\
& \\
& P^{(1)}=\frac{1}{2 \pi i} \int_{\gamma} R_{H_{\omega}}(z) U_{1} R_{H_{\omega}}(z) d z \\
& \\
& P(\varepsilon)=\frac{1}{2 \pi i} \int_{\gamma} R_{H_{\omega}}(z) U_{1} R_{H_{\omega, \varepsilon}}(z) U_{1} R_{H_{\omega}}(z) d z .
\end{aligned}
$$

Then $\left\|P^{(1)}: L^{2} \rightarrow H^{1}\right\|+\left\|P(\varepsilon): L^{2} \rightarrow H^{1}\right\|<C$ for a fixed constant if $\varepsilon \in\left[0, \varepsilon_{0}\right]$ for some $\varepsilon_{0}>0$ small enough. So, if we consider $u \in H^{1} \cap L_{c}^{2}\left(H_{\omega}\right)$ and we split $u=u_{1}+u_{2}$ with $u_{1}=P_{\varepsilon} u$, we have $u_{1}=O(\varepsilon)$ and

$$
\begin{aligned}
& \left\langle\sigma_{3} H_{\omega} u, u\right\rangle=\left\langle\sigma_{3} H_{\omega, \varepsilon} u, u\right\rangle-\varepsilon\left\langle\sigma_{3} U_{1} u, u\right\rangle=\left\langle\sigma_{3} H_{\omega, \varepsilon} u_{1}, u_{1}\right\rangle+ \\
& +\left\langle\sigma_{3} H_{\omega, \varepsilon} u_{2}, u_{2}\right\rangle-\varepsilon\left\langle\sigma_{3} U_{1} u, u\right\rangle>\left\langle\sigma_{3} H_{\omega, \varepsilon} u_{1}, u_{1}\right\rangle-\varepsilon\left\langle\sigma_{3} U_{1} u, u\right\rangle=O(\varepsilon)
\end{aligned}
$$

and so $\left\langle\sigma_{3} H_{\omega} u, u\right\rangle \geq 0$.

We consider the case $\operatorname{dim} \mathcal{V}>1$ in $\S 5$. 


\section{$\S 3$ Orbital instability of EXCIted States: THE CASE When the INTERNAL MODES ARE CLOSE TO THE CONTINUOUS SPECTRUM}

We will assume the following hypotheses.

(H4) $\beta(t) \in C^{3}(\mathbb{R}, \mathbb{R})$.

(H5) The operators $L_{+, \omega}=-\Delta+\omega-\beta\left(\phi_{\omega}^{2}\right)-2 \phi_{\omega}^{2} \beta^{\prime}\left(\phi_{\omega}^{2}\right)$ are such that ker $L_{+, \omega} \cap$ $H_{r}^{1}\left(\mathbb{R}^{3}\right)=0$ with $H_{r}^{1}$ introduced in $(\mathrm{H} 1)$.

(H6) In (1.1) the initial data are $u_{0} \in H^{k}\left(\mathbb{R}^{3}\right)$ for $k=2$ and satisfy $u_{0}(x)=u_{0}(-x)$. Let $H_{e}^{k}\left(\mathbb{R}^{3}\right)$ the space of such functions.

(H7) $\frac{d}{d \omega}\left\|\phi_{\omega}\right\|_{L^{2}\left(\mathbb{R}^{3}\right)}^{2} \neq 0$ for $\omega \in \mathcal{O}$ and $N_{g}\left(H_{\omega}\right) \cap H_{e}^{k}$ be is spanned by $\left\{\sigma_{3} \Phi_{\omega}, \partial_{\omega} \Phi_{\omega}\right\}$;

(H8) Let $H_{\omega}$ be the linearized operator around $e^{i t \omega} \phi_{\omega}$, see Section 2. Then $H_{\omega}$ has a certain number of simple positive eigenvalues with $0<\lambda_{j}(\omega)<\omega<2 \lambda_{j}(\omega)$. $H_{\omega}$ does not have other eigenvalues and $\pm \omega$ are not resonances.

(H9) For multi indexes $m=\left(m_{1}, m_{2}, \ldots\right)$ and $n=\left(n_{1}, \ldots\right)$, setting $\lambda(\omega)=\left(\lambda_{1}(\omega), \ldots\right)$ and $(m-n) \cdot \lambda=\sum\left(m_{j}-n_{j}\right) \lambda_{j}$, we have the following two non resonance hypotheses:

(i) $\quad(m-n) \cdot \lambda(\omega)=0$ implies $m=n$ if $|m| \leq 3$ and $|n| \leq 3$;

(ii) $(m-n) \cdot \lambda(\omega) \neq \omega$ for all $(m, n)$ with $|m|+|n| \leq 3$.

(H10) We assume the non degeneracy Hypothesis 3.7.

The key hypotheses are (H8), where the condition $\lambda_{j}(\omega)>\omega / 2$ is a quantitative description of what it means for the eigenvalues to be close to the continuous spectrum, and (H10), which is valid generically.

A standing wave is orbitally unstable if it is not orbitally stable. Recall the following definition:

Definition 3.1. A standing wave $e^{i \omega t} \phi_{\omega}(x)$ is orbitally stable if for any $\epsilon>0$ there is a $\delta(\epsilon)>0$ such that for any $\left\|u(0, x)-\phi_{\omega}\right\|_{H_{x}^{1}}<\delta(\epsilon)$ the corresponding solution $u(t, x)$ is globally defined and for any $t$ we have

$$
\inf _{\gamma \in \mathbb{R} \& x_{0} \in \mathbb{R}^{n}}\left\|u(t, x)-e^{i \gamma} \phi_{\omega}\left(x-x_{0}\right)\right\|_{H_{x}^{1}}<\epsilon .
$$

Remark. In the setting of even solutions of (1.1) or of solutions of the non translation invariant (1.3), we need to pick $x_{0}=0$ in the above definition.

In this section we will prove:

Theorem 3.2. Under hypotheses (H1-10) the excited states $e^{i \omega t} \phi_{\omega}(x)$ are orbitally unstable.

Remark. It is easy to manufacture examples in the spirit of [SW1-3,TY1-3,T] by considering short range Schrödinger operators $-\Delta+q(x)$ which admit a certain number of eigenvalues $-E_{0}<-E_{1}<\ldots<0$. For example, if there are only two simple eigenvalues with $E_{1} / 2<E_{0}-E_{1}<E_{1}$, then if $q(x)$ is generic the excited states originating from $E_{1}$ are unstable.

The proof of Theorem 3.2 covers the reminder of $\S 3$. We assume by absurd that the excited state $e^{i \omega_{0} t} \phi_{\omega_{0}}(x)$ is orbitally stable. We pick an arbitrarily small 
$\epsilon>0$ and we consider the associated $\delta=\delta(\epsilon)<\epsilon$. Then the representation (2.1) is valid for all $t \in \mathbb{R}$ with: $r(t, x) \in C\left(\mathbb{R}, H^{2}\left(\mathbb{R}^{3}\right)\right), R(t) \in N_{g}^{\perp}\left(H_{\omega(t)}^{*}\right)$ for all $t$, $\left|\omega_{0}-\omega(0)\right|<C \delta$ for fixed $C>0$ and $\left|\omega_{0}-\omega(t)\right|<C \epsilon$ for all $t$. Recall that there is a real valued function $F\left(|u|^{2}\right)$ with $\beta\left(|u|^{2}\right) u=\partial_{\bar{u}}\left(F\left(|u|^{2}\right)\right)$ and $F(0)=0$. For $u$ given by (2.1), we have $e^{-i \vartheta} \beta\left(|u|^{2}\right) u=\partial_{\bar{r}} F\left(\left|\phi_{\omega}+r\right|^{2}\right)$. Recall

$$
e^{-i \vartheta} \beta\left(|u|^{2}\right) u=\beta\left(\phi_{\omega}^{2}\right) \phi_{\omega}+\left(\beta\left(\phi_{\omega}^{2}\right)+\beta^{\prime}\left(\phi_{\omega(t)}^{2}\right) \phi_{\omega}^{2}\right) r+\beta^{\prime}\left(\phi_{\omega}^{2}\right) \phi_{\omega}^{2} \bar{r}-n(r, \bar{r}) .
$$

We have $\left(\beta\left(\phi_{\omega}^{2}\right)+\beta^{\prime}\left(\phi_{\omega(t)}^{2}\right) \phi_{\omega}^{2}\right) r+\beta^{\prime}\left(\phi_{\omega}^{2}\right) \phi_{\omega}^{2} \bar{r}=$

$$
\frac{1}{2} \partial_{\bar{r}}\left(\left(\beta\left(\phi_{\omega}^{2}\right)+\beta^{\prime}\left(\phi_{\omega}^{2}\right) \phi_{\omega}^{2}\right)|r|^{2}+\beta^{\prime}\left(\phi_{\omega}^{2}\right) \phi_{\omega}^{2}\left(\bar{r}^{2}+r^{2}\right)\right)
$$

Then $-n(r, \bar{r})=\partial_{\bar{r}} G(R)$ with $G(R)$ real valued, $G(0)=0$. For ${ }^{t} G^{\prime}(R)=\left(G_{r}, G_{\bar{r}}\right)$

$$
i R_{t}=H_{\omega(t)} R+\dot{\gamma}(t) \sigma_{3} R+\sigma_{3} \sigma_{1} G^{\prime}(R)+\dot{\gamma}(t) \sigma_{3} \Phi_{\omega}-i \dot{\omega}(t) \partial_{\omega} \Phi_{\omega} .
$$

The condition $R(t) \in N_{g}^{\perp}\left(H_{\omega(t)}^{*}\right)$ yields the modulation equations

$$
\begin{aligned}
& i \dot{\omega}\left\langle\Phi, \partial_{\omega} \Phi\right\rangle=\left\langle\sigma_{3} \dot{\gamma} R+N(R)+i \dot{\omega} \partial_{\omega} P_{N_{g}\left(H_{\omega}\right)} R, \Phi\right\rangle \\
& \dot{\gamma}\left\langle\Phi, \partial_{\omega} \Phi\right\rangle=-\left\langle\text { same as above, } \sigma_{3} \partial_{\omega} \Phi\right\rangle .
\end{aligned}
$$

These can be used to express $i \dot{\omega}=i \dot{\omega}(\omega, R), \dot{\gamma}=\dot{\gamma}(\omega, R)$.

Lemma 3.3. We can write with smooth functions in $r$ and $\bar{r} \in H^{2}\left(\mathbb{R}^{3}\right)$

$$
\begin{aligned}
& i \dot{\omega}=i \dot{\omega}(\omega, r, \bar{r})=\nu(r, \bar{r})-\nu(\bar{r}, r) \text { with } \overline{\nu(r, \bar{r})}=\nu(\bar{r}, r) \\
& \dot{\gamma}=\dot{\gamma}(\omega, r, \bar{r})=\mu(r, \bar{r})+\mu(\bar{r}, r) \text { with } \overline{\mu(r, \bar{r})}=\mu(\bar{r}, r) .
\end{aligned}
$$

Proof. Let $P_{N_{g}\left(H_{\omega}\right)}$ be the projection onto $N_{g}\left(H_{\omega}\right)$ in $L^{2}=N_{g}\left(H_{\omega}\right) \oplus N_{g}^{\perp}\left(H_{\omega}^{*}\right)$. We apply $P_{N_{g}\left(H_{\omega}\right)}$ to $(2.2)$ obtaining

$$
i P_{N_{g}\left(H_{\omega}\right)} R_{t}-\dot{\gamma}(t) P_{N_{g}\left(H_{\omega}\right)} \sigma_{3} R-\sigma_{3} \dot{\gamma}(t) \Phi_{\omega}+i \dot{\omega}(t) \partial_{\omega} \Phi_{\omega}+P_{N_{g}\left(H_{\omega}\right)} N(R) .
$$

Set $q(\omega)=\left\|\phi_{\omega}\right\|_{2}^{2}$ and $q^{\prime}(\omega)=d q(\omega) / d \omega$. Then we have

$$
P_{N_{g}\left(H_{\omega}\right)}=\sigma_{3} \Phi_{\omega}\left\langle\quad, \sigma_{3} \partial_{\omega} \Phi_{\omega}\right\rangle / q(\omega)+\partial_{\omega} \Phi_{\omega}\left\langle\quad, \Phi_{\omega}\right\rangle / q(\omega) .
$$

By $P_{N_{g}\left(H_{\omega}\right)} R=0$, which implies $P_{N_{g}\left(H_{\omega}\right)} R_{t}=-\dot{\omega} \partial_{\omega} P_{N_{g}\left(H_{\omega}\right)} R$, we get

$$
\left(q^{\prime}(\omega)+\left[\begin{array}{cc}
-\left\langle\partial_{\omega} P_{N_{g}\left(H_{\omega}\right)} R, \Phi_{\omega}\right\rangle & \left\langle\sigma_{3} R, \Phi_{\omega}\right\rangle \\
-\left\langle\partial_{\omega} P_{N_{g}\left(H_{\omega}\right)} R, \sigma_{3} \partial_{\omega} \Phi_{\omega}\right\rangle & \left\langle R, \partial_{\omega} \Phi_{\omega}\right\rangle
\end{array}\right]\right)\left[\begin{array}{c}
i \dot{\omega} \\
-\dot{\gamma}
\end{array}\right]=\left[\begin{array}{c}
\left\langle N(R), \Phi_{\omega}\right\rangle \\
\left\langle N(R), \sigma_{3} \partial_{\omega} \Phi_{\omega}\right\rangle
\end{array}\right] .
$$


By an elementary computation we have

$$
\begin{aligned}
& \left\langle\partial_{\omega} P_{N_{g}\left(H_{\omega}\right)} R, \Phi_{\omega}\right\rangle=\left\langle r+\bar{r}, \frac{\left\langle\partial_{\omega}^{2} \Phi, \Phi\right\rangle}{q^{\prime}} \phi_{\omega}+q^{\prime} \partial_{\omega} \frac{\phi_{\omega}}{q^{\prime}(\omega)}\right\rangle \\
& \left\langle\partial_{\omega} P_{N_{g}\left(H_{\omega}\right)} R, \sigma_{3} \partial_{\omega} \Phi_{\omega}\right\rangle=\left\langle r-\bar{r}, \frac{\left\|\partial_{\omega} \Phi\right\|_{2}^{2}}{q^{\prime}} \partial_{\omega} \phi_{\omega}+q^{\prime} \partial_{\omega} \frac{\partial_{\omega} \phi_{\omega}}{q^{\prime}(\omega)}\right\rangle
\end{aligned}
$$

and so, for some real valued exponentially decreasing functions $\alpha(\omega, x)$ and $\beta(\omega, x)$, we have the following, which yields (1):

$$
\left(q^{\prime}(\omega)+\left[\begin{array}{cc}
\langle r+\bar{r}, \alpha(\omega)\rangle & \left\langle r-\bar{r}, \phi_{\omega}\right\rangle \\
\langle r-\bar{r}, \beta(\omega)\rangle & \left\langle r+\bar{r}, \partial_{\omega} \phi_{\omega}\right\rangle
\end{array}\right]\right)\left[\begin{array}{c}
i \dot{\omega} \\
-\dot{\gamma}
\end{array}\right]=\left[\begin{array}{c}
\left\langle n(r, \bar{r})-n(\bar{r}, r), \phi_{\omega}\right\rangle \\
\left\langle n(r, \bar{r})+n(\bar{r}, r), \partial_{\omega} \phi_{\omega}\right\rangle
\end{array}\right] .
$$

The regularity of $\mu(r, \bar{r})$ and $\nu(r, \bar{r})$ follows by the smoothness of $n(z, \bar{z})$ as a function in $z \in \mathbb{C}$, and by the fact that $H^{2}\left(\mathbb{R}^{3}\right)$ is an algebra. This completes Lemma 3.3.

For each $j$ we consider a generator $\xi_{j} \in \operatorname{ker}\left(H_{\omega}-\lambda_{j}\right)$ such that $\left\langle\xi_{j}, \sigma_{3} \xi_{j}\right\rangle=s_{j}$ with $s_{j}=1$ (resp. $s_{j}=-1$ ) if $\lambda_{j}$ has positive (resp. negative) signature. Since $e^{i \omega t} \phi_{\omega}$ is an excited state, by Theorem 1.1 at least for one $j$ we have $s_{j}=-1$, so in particular we can assume $s_{1}=-1$. Indeed under hypothesis (H8), if $\xi$ is a generator of $\operatorname{ker}\left(H_{\omega}-\lambda_{j}\right)$ for any $j$, then $\left\langle\xi, \sigma_{3} \xi\right\rangle \neq 0$. We expand $R(t) \in N_{g}^{\perp}\left(H_{\omega(t)}^{*}\right)$ into

$$
R(t)=\left(z \cdot \xi+\bar{z} \cdot \sigma_{1} \xi\right)+f(t) \in\left[\sum_{j, \pm} \operatorname{ker}\left(H_{\omega(t)} \mp \lambda_{j}(\omega(t))\right)\right] \oplus L_{c}^{2}\left(H_{\omega(t)}\right)
$$

Correspondingly we express (3.1) as

$$
\begin{aligned}
i \dot{z}_{j} \xi_{j}-\lambda_{j}(\omega) z_{j} \xi_{j} & =P_{\operatorname{ker}\left(H_{\omega}-\lambda_{j}\right)}\left(\dot{\gamma}(\omega, R) \sigma_{3} R+\sigma_{3} \sigma_{1} G^{\prime}(R)\right. \\
& \left.-i z_{j} \dot{\omega}(\omega, R) \partial_{\omega} \xi_{j}+i \dot{\omega}(\omega, R) \partial_{\omega} P_{\operatorname{ker}\left(H_{\omega}-\lambda_{j}\right)} R\right) \\
i P_{c}\left(H_{\omega}\right) \dot{f}-H_{\omega} f & =P_{c}\left(H_{\omega}\right)\left(\dot{\gamma}(\omega, R) \sigma_{3} R+\sigma_{3} \sigma_{1} G^{\prime}(R)+i \dot{\omega}(\omega, R) \partial_{\omega} P_{c}\left(H_{\omega}\right) R\right) .
\end{aligned}
$$

We use the multi index notation $z^{m}=\prod_{j} z_{j}^{m_{j}}$. We consider the expansion

$$
\sigma_{3} \sigma_{1} G^{\prime}(R)=\sum_{|m+n|=2}^{3} R_{m, n}(\omega) z^{m} \bar{z}^{n}+\sum_{|m+n|=1} z^{m} \bar{z}^{n} A_{m, n}(\omega) f+O\left(f^{2}\right)+\cdots
$$

with $R_{m, n}(\omega, x)$ and $A_{m, n}(\omega, x)$ real vectors and matrices exponentially decreasing in $x$. We have

$$
A_{m, n}(\omega)=\frac{\sigma_{3} \sigma_{1}}{m ! n !} \partial_{z}^{m} \partial_{\bar{z}}^{n} \partial_{f} G^{\prime}(0), \quad R_{m, n}(\omega)=\frac{\sigma_{3} \sigma_{1}}{m ! n !} \partial_{z}^{m} \partial_{\bar{z}}^{n} G^{\prime}(0) .
$$


Notice $A_{m, n}(\omega)=-\sigma_{1} A_{n, m}(\omega) \sigma_{1}$ and $\sigma_{1} R_{m, n}(\omega)=-R_{n, m}(\omega)$. Indeed by definition $\sigma_{1} R=\bar{R}$, from which we get $\sigma_{1} f=\bar{f}$ and $\sigma_{1} G^{\prime}(R)=\overline{G^{\prime}(R)}$. We also have $\sigma_{1} H_{\omega}=-H_{\omega} \sigma_{1}$. Then, taking complex conjugate of (3.1) and applying to the resulting equation $\sigma_{1}$, we get

$$
\begin{aligned}
& -i \dot{R}+H_{\omega} R=. . \sigma_{1} R_{m, n}(\omega) z^{n} \bar{z}^{m}+. . \bar{z}^{m} z^{n} \sigma_{1} A_{m, n}(\omega) \sigma_{1} f+. . \\
& =. .-R_{n, m}(\omega) z^{n} \bar{z}^{m}+. .-\bar{z}^{m} z^{n} A_{n, m}(\omega) f+. .
\end{aligned}
$$

which yields $A_{m, n}(\omega)=-\sigma_{1} A_{n, m}(\omega) \sigma_{1}$ and $\sigma_{1} R_{m, n}(\omega)=-R_{n, m}(\omega)$. We set $\delta_{j}=$ $\left(\delta_{j 1}, \delta_{j 2}, \ldots\right)$ with $\delta_{j k}$ the Kronecker delta. We have

$$
A_{\delta_{\ell}, 0}(\omega)=\sigma_{3} \sigma_{1} \partial_{z_{\ell}} \partial_{f} G^{\prime}(0)=\sigma_{3} \sigma_{1} \partial_{z_{\ell}} \partial_{f} G^{\prime}(0)=\sigma_{3} \sigma_{1} G^{(3)}(0)\left(\quad, \xi_{\ell}, P_{c}\left(H_{\omega}\right)\right)
$$

where $G^{(3)}(0)$ is written as a symmetric trilinear form and where one of the vectors of the triple is $\xi_{\ell}$. We have

$$
\begin{aligned}
& P_{c}\left(H_{\omega}\right) R_{\delta_{j}+\delta_{\ell}, 0}(\omega)=\frac{\sigma_{3} \sigma_{1}}{\left(\delta_{j}+\delta_{\ell}\right) !} P_{c}\left(H_{\omega}^{*}\right) \partial_{z_{j}} \partial_{z_{\ell}} G^{\prime}(0) \\
& =\frac{\sigma_{3} \sigma_{1}}{\left(\delta_{j}+\delta_{\ell}\right) !} \partial_{z_{j}} \partial_{z_{\ell}} G^{\prime}(0) \circ P_{c}\left(H_{\omega}\right)=\frac{\sigma_{3} \sigma_{1}}{\left(\delta_{j}+\delta_{\ell}\right) !} G^{(3)}(0)\left(\xi_{j}, \xi_{\ell}, P_{c}\left(H_{\omega}\right)\right) .
\end{aligned}
$$

For later use we record:

Lemma 3.4. For $\lambda_{j}=\lambda_{j}(\omega), \xi_{j}=\xi_{j}(\omega), A_{0, \delta_{\ell}}=A_{0, \delta_{\ell}}(\omega)$ and $R_{\delta_{j}+\delta_{\ell}, 0}=$ $R_{\delta_{j}+\delta_{\ell}, 0}(\omega)$ we have

$$
\begin{aligned}
& \left\langle A_{0, \delta_{\ell}} R_{H_{\omega}}^{+}\left(\lambda_{j}+\lambda_{\ell}\right) R_{\delta_{j}+\delta_{\ell}, 0}, \sigma_{3} \xi_{j}\right\rangle=\frac{1}{\left(\delta_{j}+\delta_{\ell}\right) !} \times \\
& \left\langle R_{H_{\omega}}^{+}\left(\lambda_{j}+\lambda_{\ell}\right) \sigma_{3} \sigma_{1} G^{(3)}(0)\left(\xi_{j}, \xi_{\ell}, P_{c}\left(H_{\omega}\right)\right), \sigma_{3} \sigma_{3} \sigma_{1} G^{(3)}(0)\left(\xi_{j}, \xi_{\ell}, P_{c}\left(H_{\omega}\right)\right)\right\rangle .
\end{aligned}
$$

Taking the imaginary part of the above formula, we have

$$
\begin{aligned}
& \Im\left\langle A_{0, \delta_{\ell}} R_{H_{\omega}}^{+}\left(\lambda_{j}+\lambda_{\ell}\right) R_{\delta_{j}+\delta_{\ell}, 0}(\omega), \sigma_{3} \xi_{j}(\omega)\right\rangle=\frac{\pi}{\left(\delta_{j}+\delta_{\ell}\right) !} \times \\
& \left\langle\delta\left(H_{\omega}-\lambda_{j}-\lambda_{\ell}\right) \sigma_{3} \sigma_{1} G^{(3)}(0)\left(\xi_{j}, \xi_{\ell}, P_{c}\left(H_{\omega}\right)\right), \sigma_{3} \sigma_{3} \sigma_{1} G^{(3)}(0)\left(\xi_{j}, \xi_{\ell}, P_{c}\left(H_{\omega}\right)\right)\right\rangle \\
& \geq 0 \text { for any } G^{(3)}(0)\left(\xi_{j}, \xi_{\ell}, P_{c}\left(H_{\omega}\right)\right) .
\end{aligned}
$$

Proof. We recall that $G^{(3)}(0)\left(\xi_{j}, \xi_{\ell}, P_{c}\left(H_{\omega}\right)\right) \in L^{2}$ is defined by the equality $\left\langle g, G^{(3)}(0)\left(\xi_{j}, \xi_{\ell}, P_{c}\left(H_{\omega}\right)\right)\right\rangle=\frac{d}{d t} G^{(2)}\left(t P_{c}\left(H_{\omega}\right) g\right)_{t=0}\left(\xi_{j}, \xi_{\ell}\right)$. We assume the first 
formula and we set $F=\sigma_{3} \sigma_{1} G^{(3)}(0)\left(\xi_{j}, \xi_{\ell}, P_{c}\left(H_{\omega}\right)\right)$. Recall there are isomorphisms $W\left(H_{\omega}\right): L^{p} \rightarrow L_{c}^{p}\left(H_{\omega}\right)$ and $Z\left(H_{\omega}\right)$ its inverse, such that $P_{c}\left(H_{\omega}\right) H_{\omega}=$ $W\left(H_{\omega}\right) \sigma_{3}(-\Delta+\omega) Z\left(H_{\omega}\right)$, [Cu2, CPV]. Furthermore, from the definitions one gets $W^{*}\left(H_{\omega}\right) \sigma_{3}=\sigma_{3} Z\left(H_{\omega}\right)$, see $[\mathrm{Cu} 2, \mathrm{CPV}]$. For $P_{c}\left(H_{\omega}\right) F=W\left(H_{\omega}\right) \widetilde{F}$ we have

$$
\begin{aligned}
& \left\langle R_{H_{\omega}}^{+}\left(\lambda_{j}+\lambda_{\ell}\right) F, \sigma_{3} F\right\rangle=\left\langle R_{H_{\omega}}^{+}\left(\lambda_{j}+\lambda_{\ell}\right) W\left(H_{\omega}\right) \widetilde{F}, \sigma_{3} W\left(H_{\omega}\right) \widetilde{F}\right\rangle= \\
= & \left\langle R_{\sigma_{3}(-\Delta+\omega)}^{+}\left(\lambda_{j}+\lambda_{\ell}\right) \widetilde{F}, W^{*}\left(H_{\omega}\right) \sigma_{3} W\left(H_{\omega}\right) \widetilde{F}\right\rangle= \\
= & \left\langle R_{\sigma_{3}(-\Delta+\omega)}^{+}\left(\lambda_{j}+\lambda_{\ell}\right) \widetilde{F}, \sigma_{3} Z\left(H_{\omega}\right) W\left(H_{\omega}\right) \widetilde{F}\right\rangle=\left\langle R_{\sigma_{3}(-\Delta+\omega)}^{+}\left(\lambda_{j}+\lambda_{\ell}\right) \widetilde{F}, \sigma_{3} \widetilde{F}\right\rangle
\end{aligned}
$$

Let now $\widetilde{F}_{1}$ and $\widetilde{F}_{2}$ be the two components of the vector $\widetilde{F}$. Then

$$
\begin{aligned}
& \Im\left\langle R_{\sigma_{3}(-\Delta+\omega)}^{+}\left(\lambda_{j}+\lambda_{\ell}\right) \widetilde{F}, \sigma_{3} \widetilde{F}\right\rangle=\pi\left\langle\delta\left(\sigma_{3}(-\Delta+\omega)-\lambda_{j}-\lambda_{\ell}\right) \widetilde{F}, \sigma_{3} \widetilde{F}\right\rangle \\
= & \pi\left\langle\delta\left(-\Delta+\omega-\lambda_{j}-\lambda_{\ell}\right) \widetilde{F}_{1}, \widetilde{F}_{1}\right\rangle-\pi\left\langle\delta\left(\sigma \Delta-\omega-\lambda_{j}-\lambda_{\ell}\right) \widetilde{F}_{2}, \widetilde{F}_{2}\right\rangle .
\end{aligned}
$$

We have $\left\langle\delta\left(\Delta-\omega-\lambda_{j}-\lambda_{\ell}\right) \widetilde{F}_{2}, \widetilde{F}_{2}\right\rangle=0$ for any $\widetilde{F}_{2}$. Notice that in our hypothesis we have $\lambda_{j}+\lambda_{\ell}>\omega$. Setting $\rho_{0}=\sqrt{\lambda_{j}+\lambda_{\ell}-\omega}$ we have for any $\widetilde{F}_{1} \in H^{s}$ for $s>1 / 2$

$$
\left\langle\delta\left(-\Delta+\omega-\lambda_{j}-\lambda_{\ell}\right) \widetilde{F}_{1}, \widetilde{F}_{1}\right\rangle=\frac{1}{2 \rho_{0}} \int_{|\eta|=\rho_{0}}\left|\widehat{\widetilde{F}}_{1}(\eta)\right|^{2} d \sigma(\eta) \geq 0 .
$$

We prove now the first formula in the statement. We have

$$
\begin{aligned}
& \left\langle A_{0, \delta_{\ell}} R_{H_{\omega}}^{+}\left(\lambda_{j}+\lambda_{\ell}\right) R_{\delta_{j}+\delta_{\ell}, 0}, \sigma_{3} \xi_{j}\right\rangle=-\left\langle\sigma_{1} A_{\delta_{\ell}, 0} \sigma_{1} R_{H_{\omega}}^{+}\left(\lambda_{j}+\lambda_{\ell}\right) R_{\delta_{j}+\delta_{\ell}, 0}, \sigma_{3} \xi_{j}\right\rangle= \\
& =-\left\langle\sigma_{1} \sigma_{3} \sigma_{1} \partial_{z_{\ell}} \partial_{f} G^{\prime}(0) \sigma_{1} R_{H_{\omega}}^{+}\left(\lambda_{j}+\lambda_{\ell}\right) R_{\delta_{j}+\delta_{\ell}, 0}, \sigma_{3} \xi_{j}\right\rangle= \\
& =\left\langle\partial_{z_{\ell}} \partial_{f} G^{\prime}(0) \sigma_{1} R_{H_{\omega}}^{+}\left(\lambda_{j}+\lambda_{\ell}\right) R_{\delta_{j}+\delta_{\ell}, 0}, \xi_{j}\right\rangle \\
& =\left\langle\sigma_{1} R_{H_{\omega}}^{+}\left(\lambda_{j}+\lambda_{\ell}\right) R_{\delta_{j}+\delta_{\ell}, 0}, G^{(3)}(0)\left(\xi_{j}, \xi_{\ell}, P_{c}\left(H_{\omega}\right)\right)\right\rangle= \\
& =\frac{1}{\left(\delta_{j}+\delta_{\ell}\right) !}\left\langle\sigma_{1} R_{H_{\omega}}^{+}\left(\lambda_{j}+\lambda_{\ell}\right) \sigma_{3} \sigma_{1} G^{(3)}(0)\left(\xi_{j}, \xi_{\ell}, P_{c}\left(H_{\omega}\right)\right), G^{(3)}(0)\left(\xi_{j}, \xi_{\ell}, P_{c}\left(H_{\omega}\right)\right)\right\rangle \\
& =\frac{1}{\left(\delta_{j}+\delta_{\ell}\right) !}\left\langle R_{H_{\omega}}^{+}\left(\lambda_{j}+\lambda_{\ell}\right) h_{j, \ell}, \sigma_{3} h_{j, \ell}\right\rangle \text { where } h_{j, \ell}=\sigma_{3} \sigma_{1} G^{(3)}(0)\left(\xi_{j}, \xi_{\ell}, P_{c}\left(H_{\omega}\right)\right) .
\end{aligned}
$$

This concludes the proof of Lemma 3.4.

For $O_{l o c}\left(z^{n}\right)=\sum_{\ell} O_{l o c}\left(\left|z_{\ell}^{n}\right|\right),(3.4)$ can be expressed as

$$
\begin{aligned}
& i f_{t}=\left(H_{\omega(t)}+P_{c}\left(H_{\omega}\right) \sigma_{3} \dot{\gamma}\right) f+\sum_{|m+n|=2} z^{m} \bar{z}^{n} P_{c}\left(H_{\omega}\right) R_{m, n}(\omega) \\
& +\sum_{|m+n|=1} z^{m} \bar{z}^{n} P_{c}\left(H_{\omega}\right) A_{m, n}(\omega) f+O\left(f^{2}\right)+O_{l o c}\left(z^{3}\right)
\end{aligned}
$$


and

$$
\begin{aligned}
i \dot{z}_{j} \xi_{j}-\lambda_{j}(\omega) z_{j} \xi_{j} & =P_{\operatorname{ker}\left(H_{\omega}-\lambda_{j}\right)}\left(\sum_{|m+n|=2} z^{m} \bar{z}^{n} R_{m, n}(\omega)+\sum_{|m+n|=3} z^{m} \bar{z}^{n} R_{m, n}^{(1)}(\omega)\right. \\
& +\sum_{|m+n|=1} z^{m} \bar{z}^{n} A_{m, n}(\omega) f+O\left(f^{2}\right)+O_{l o c}\left(z^{4}\right)
\end{aligned}
$$

where in (3.7) the coefficients $A_{m, n}(\omega)$ and $R_{m, n}(\omega)$ are those of the expansion of $\sigma_{3} \sigma_{1} G^{\prime}(R)$ in $(3.5)$ and where $R_{m, n}^{(1)}(\omega)$ are real and exponentially decreasing vectors. We have:

Lemma 3.5. Let $0<\delta<\epsilon$ be as in the Definition 3.1 of linear stability. For any $C_{1}>0$ there are a $\varepsilon\left(C_{1}\right)>0$ and a $C\left(C_{1}\right)$ such that if, for $0<\varepsilon<\varepsilon\left(C_{1}\right)$, we have $\left\|z_{j}\right\|_{L^{4}(0, T)}^{2} \leq C_{1} \varepsilon$ for all $j$, then for all admissible pairs $(p, q)$ we have for a fixed $c_{0}$

$$
\|f\|_{L_{t}^{p}\left((0, T), W_{x}^{1, q}\right)}<c_{0} \delta+C\left(C_{1}\right) \varepsilon .
$$

Proof. In this proof $P_{c}(\omega)=P_{c}\left(H_{\omega}\right)$. We split $P_{c}(\omega)=P_{+}(\omega)+P_{-}(\omega)$, with $P_{ \pm}(\omega)$ the spectral projections in $\mathbb{R}_{ \pm} \cap \sigma_{c}\left(H_{\omega}\right)$, see [Cu3]. By orbital stability we can fix $\omega_{0}$ such that $\left|\omega(t)-\omega_{0}\right|=O(\epsilon)$ for all $t$. We write following [BP]

$$
\begin{aligned}
& i f_{t}=\left\{H_{\omega_{0}}+\left(\dot{\gamma}+\omega-\omega_{0}\right)\left(P_{+}\left(\omega_{0}\right)-P_{-}\left(\omega_{0}\right)\right)\right\} P_{c}\left(\omega_{0}\right) f \\
& +O_{l o c}(\epsilon f)+O\left(f^{2}\right)+O_{l o c}\left(z^{2}\right) \\
& \text { where } O_{l o c}(\epsilon f)=\left(\dot{\gamma}+\omega-\omega_{0}\right)\left(P_{c}\left(\omega_{0}\right) \sigma_{3}-\left(P_{+}\left(\omega_{0}\right)-P_{-}\left(\omega_{0}\right)\right)\right) f \\
& +\left(V(\omega)-V\left(\omega_{0}\right)\right) f+\left(\dot{\gamma}+\omega-\omega_{0}\right)\left(P_{c}(\omega)-P_{c}\left(\omega_{0}\right)\right) \sigma_{3} f .
\end{aligned}
$$

To justify the notation $O_{l o c}(\epsilon f)$ we notice that $\omega-\omega_{0}=O(\epsilon)$ and the following fact: $\forall p \in[1,2] q \in[2, \infty)$ with $c_{p, q}(\omega)$ upper semicontinuous in $\omega$, [Cu3,Cu1],

$$
\left\|P_{c}(\omega) \sigma_{3}-\left(P_{+}(\omega)-P_{-}(\omega)\right): L^{q} \rightarrow L^{p}\right\| \leq c_{p, q}(\omega)<\infty .
$$

By $[\mathrm{Cu} 2, \mathrm{CPV}] P_{c}(\omega) e^{-i t H_{\omega}}$ satisfies for any fixed $\omega \in \mathcal{O}$ the Stricharz estimates, i.e. there is a $C(\omega, k)$ upper semicontinuous in $\omega$ such that for all admissible pairs $(p, q)$ and $(a, b)$ and we have

$$
\begin{aligned}
& \left\|P_{c}(\omega) e^{-i t H_{\omega}} \varphi(x)\right\|_{L_{t}^{p} W_{x}^{k, q}} \leq C(\omega)\|\varphi(x)\|_{H_{x}^{k}} \\
& \left\|P_{c}(\omega) e^{-i t H_{\omega}} \psi\right\|_{L_{t}^{p} W_{x}^{k, q}} \leq C(\omega)\|\psi(t, x)\|_{L_{t}^{a^{\prime}} W_{x}^{k, b^{\prime}}} .
\end{aligned}
$$

We have $\|f\|_{L_{t}^{p} W_{x}^{k, q}} \approx\left\|P_{c}\left(\omega_{0}\right) f\right\|_{L_{t}^{p} W_{x}^{k, q}}$ by 


$$
\begin{aligned}
& \|f\|_{L_{t}^{p} W_{x}^{k, q}}=\left\|P_{c}\left(\omega_{0}\right) f\right\|_{L_{t}^{p} W_{x}^{k, q}}+\left\|\left(P_{c}(\omega)-P_{c}\left(\omega_{0}\right)\right) f\right\|_{L_{t}^{p} W_{x}^{k, q}} \\
& =\left\|P_{c}\left(\omega_{0}\right) f\right\|_{L_{t}^{p} W_{x}^{k, q}}+O(\epsilon)\|f\|_{L_{t}^{p} W_{x}^{k, q}}
\end{aligned}
$$

For $U_{ \pm}\left(t, t^{\prime}\right)=e^{-i\left(t-t^{\prime}\right) H_{\omega_{0}}} e^{ \pm i \int_{t^{\prime}}^{t} d \tau\left(\dot{\gamma}(\tau)+\omega(\tau)-\omega_{0}\right)} P_{ \pm}\left(\omega_{0}\right)$, we have

$$
P_{ \pm}\left(\omega_{0}\right) f(t)=U_{ \pm}(t, 0) f(0)+\int_{0}^{t} U_{ \pm}\left(t, t^{\prime}\right)\left(O_{l o c}(\epsilon f)+O\left(f^{2}\right)+O_{l o c}\left(z^{2}\right)\right) d t^{\prime} .
$$

Since $\|f(0)\|_{H^{1}}<\delta$, there is a $T_{1} \in(0, T]$ such that (3.8) is true in $\left(0, T_{1}\right)$. Using the Stricharz estimates (3.10), in particular the "endpoint Stricharz estimate, in $\left(0, T_{1}\right)$ we have

$$
\begin{aligned}
& \|f\|_{L_{t}^{p} W_{x}^{k, q}} \approx\left\|P_{c}\left(\omega_{0}\right) f\right\|_{L_{t}^{p} W_{x}^{k, q}} \\
& \leq C(\omega) \delta+C(\omega) \epsilon\|f\|_{L_{t}^{2} W_{x}^{k, 6}}+C(\omega) \epsilon O\left(\delta^{2}+\varepsilon^{2}\right)+C(\omega) C_{1} \varepsilon .
\end{aligned}
$$

Since $\epsilon>0$ is small, we conclude

$$
\|f\|_{L_{t}^{p} W_{x}^{k, q}} \leq 2 C(\omega) \delta+2 C(\omega) \epsilon O\left(\delta^{2}+\varepsilon^{2}\right)+2 C(\omega) C_{1} \varepsilon .
$$

Then by a continuity argument, we conclude that (3.8) holds in $\left(0, T_{1}\right)$ with $T_{1}=T$, i.e. the claim of Lemma 3.5.

Having obtained Lemma 3.5, we rewrite (3.9) in more precise form:

$$
\begin{aligned}
& i f_{t}=\left\{H_{\omega_{0}}+\left(\dot{\gamma}+\omega-\omega_{0}\right)\left(P_{+}\left(\omega_{0}\right)-P_{-}\left(\omega_{0}\right)\right)\right\} f \\
& +\sum_{|m+n|=2} z^{m} \bar{z}^{n} P_{c}\left(\omega_{0}\right) R_{m, n}(\omega)+\sum_{|m+n|=1} z^{m} \bar{z}^{n} P_{c}\left(\omega_{0}\right) A_{m, n}(\omega) f+ \\
& +\left(\dot{\gamma}+\omega-\omega_{0}\right)\left(P_{c}\left(\omega_{0}\right) \sigma_{3}-\left(P_{+}\left(\omega_{0}\right)-P_{-}\left(\omega_{0}\right)\right)\right) f+\left(V(\omega)-V\left(\omega_{0}\right)\right) f \\
& +\left(\dot{\gamma}+\omega-\omega_{0}\right)\left(P_{c}(\omega)-P_{c}\left(\omega_{0}\right)\right) \sigma_{3} f+O\left(f^{2}\right)+O_{l o c}\left(z^{3}\right) \\
& +\left(P_{c}(\omega)-P_{c}\left(\omega_{0}\right)\right)\left(\sum_{|m+n|=2} z^{m} \bar{z}^{n} R_{m, n}(\omega)+\sum_{|m+n|=1} z^{m} \bar{z}^{n} A_{m, n}(\omega) f\right) .
\end{aligned}
$$

We then set

$$
f_{2}=f+\sum_{|m+n|=2} R_{H_{\omega_{0}}}^{+}\left((m-n) \cdot \lambda\left(\omega_{0}\right)\right) P_{c}\left(H_{\omega_{0}}\right) R_{m, n}(\omega) z^{m} \bar{z}^{n} .
$$

We will need below: 
Lemma 3.6. Assume the hypotheses of Lemma 3.5. Then for $s>1$ sufficiently large we can decompose $f_{2}=h_{1}+h_{2}+h_{3}+h_{4}$ with:

(1) for a fixed $c_{0}\left(\omega_{0}\right),\left\|h_{1}\right\|_{L_{t}^{2}\left(\mathbb{R}, L_{x}^{2,-s}\right)} \leq c_{0}\left(\omega_{0}\right)\|f(0)\|_{H^{1}} \leq c_{0}\left(\omega_{0}\right) \delta$;

(2) for a fixed $c_{1}\left(\omega_{0}\right),\left\|h_{2}\right\|_{L_{t}^{2}\left(\mathbb{R}, L_{x}^{2,-s}\right)} \leq c_{1}\left(\omega_{0}\right)|z(0)|^{2} \leq c_{1}\left(\omega_{0}\right) \delta^{2}$;

(3) $\left\|h_{3}\right\|_{L_{t}^{2}\left((0, T), L_{x}^{2,-s}\right)} \leq O(\epsilon(\varepsilon+\delta))$;

(4) for all admissible pairs $(r, p)$ we have $\left\|h_{4}\right\|_{L_{t}^{r}\left((0, T), L_{x}^{p}\right)}=O(\epsilon \varepsilon)$. All the constants, included those in the big $O$ 's, do not depend on $T$.

Proof. The proof is basically that in $\S 4[\mathrm{CM}]$. We have schematically

$$
\begin{aligned}
& i \partial_{t} P_{c}\left(H_{\omega_{0}}\right) f_{2}=\left(H_{\omega_{0}}+\left(\dot{\gamma}+\omega-\omega_{0}\right)\left(P_{+}\left(\omega_{0}\right)-P_{-}\left(\omega_{0}\right)\right)\right) P_{c}\left(H_{\omega_{0}}\right) f_{2}+ \\
& +\sum_{|m|=2} O\left(|z|^{3}\right)\left(R_{H_{\omega_{0}}}^{+}\left(m \cdot \lambda\left(\omega_{0}\right)\right) R_{m, 0}\left(\omega_{0}\right)+R_{H_{\omega_{0}}}^{+}\left(-m \cdot \lambda\left(\omega_{0}\right)\right) R_{0, m}\left(\omega_{0}\right)\right) \\
& +P_{c}\left(H_{\omega_{0}}\right)\left(o(1) O_{l o c}\left(|z|^{2}\right)+o(1) O_{l o c}(f)+O\left(f^{2}\right)\right) .
\end{aligned}
$$

For $h_{1}(0)=f(0)$ let

$i \partial_{t}\left(h_{1}+h_{2}\right)=\left(H_{\omega_{0}}+\left(\dot{\gamma}+\omega-\omega_{0}\right)\left(P_{+}-P_{-}\right)\right)\left(h_{1}+h_{2}\right), \quad h_{1}(0)+h_{2}(0)=f_{2}(0)$.

Then (1) follows by Stricharz estimates applied to $P_{ \pm}\left(\omega_{0}\right) h_{1}(t)=U_{ \pm}(t, 0) f(0)$, with $U_{ \pm}(t, s)$ defined in Lemma 3.5. To get (2) recall from [Cu3] that for a constant $C=C\left(\Lambda, \omega_{0}\right)$ upper semicontinuous in $\omega_{0}$ and in $\Lambda>\omega$ we have

$$
\left\|U_{ \pm}\left(t, t^{\prime}\right) R_{H_{\omega}}^{+}(\Lambda) P_{c} g\right\|_{L_{x}^{2,-s}}<C\left\langle t-t^{\prime}\right\rangle^{-\frac{3}{2}}\|g\|_{L_{x}^{2, s}}, s>s_{0} .
$$

By $f_{2}(0)=\sum_{|m+n|=2} R_{H_{\omega_{0}}}^{+}\left((m-n) \lambda\left(\omega_{0}\right)\right) R_{m, n}\left(\omega_{0}\right) z^{m}(0) \bar{z}^{n}(0)$ and (5) we get (2). Next we define $h_{3}(0)=0$ and

$$
\begin{aligned}
& i \partial_{t} P_{c}\left(H_{\omega_{0}}\right) h_{3}=\left(H_{\omega_{0}}+\left(\dot{\gamma}+\omega-\omega_{0}\right)\left(P_{+}\left(\omega_{0}\right)-P_{-}\left(\omega_{0}\right)\right)\right) P_{c}\left(H_{\omega_{0}}\right) h_{3}+ \\
& +P_{c}\left(H_{\omega_{0}}\right)\left(O(\epsilon) O_{l o c}\left(|z|^{2}\right)+O(\epsilon) O_{l o c}(f)+O\left(f^{2}\right)\right) .
\end{aligned}
$$

Then (3) follows in a standard way from Strichartz inequalities, see [CM]. Finally we set $h_{4}(0)=0$ and

$$
\begin{aligned}
& i \partial_{t} h_{4}=\left(H_{\omega_{0}}+\left(\dot{\gamma}+\omega-\omega_{0}\right)\left(P_{+}-P_{-}\right)\right) h_{4}+ \\
& +\sum_{|m|=2} O\left(|z|^{3}\right)\left(R_{H_{\omega_{0}}}^{+}\left(m \cdot \lambda\left(\omega_{0}\right)\right) R_{m, 0}\left(\omega_{0}\right)+R_{H_{\omega_{0}}}^{+}\left(-m \cdot \lambda\left(\omega_{0}\right)\right) R_{0, m}\left(\omega_{0}\right)\right) .
\end{aligned}
$$

Then we have $h_{4}=h_{41}+h_{42}$ with $h_{4 j}=\sum_{ \pm} h_{4 j \pm}$ with 


$$
h_{41 \pm}(t)=\sum_{|m|=2} \int_{0}^{t} U_{ \pm}\left(t, t^{\prime}\right) O\left(|z(s)|^{3}\right) R_{H_{\omega_{0}}}^{+}\left(m \cdot \lambda\left(\omega_{0}\right)\right) R_{m, 0}\left(\omega_{0}\right) d t^{\prime}
$$

and $h_{42 \pm}$ defined similarly. By (5) we get $\left\|h_{4 j \pm}(t)\right\|_{L_{x}^{2,-s}} \leq C \epsilon \int_{0}^{t}\left\langle t-t^{\prime}\right\rangle^{-\frac{3}{2}}\left|z\left(t^{\prime}\right)\right|^{2} d t^{\prime}$ and so $\left\|h_{4}\right\|_{L_{t}^{2} L_{x}^{2,-s}} \leq \epsilon\|z\|_{L_{t}^{4}}^{2}=O(\epsilon \varepsilon)$. This concludes the proof of Lemma 3.6.

By substitution of (3.12) in the discrete part in (3.7) we get

$$
\begin{aligned}
& i \dot{z}_{j} \xi_{j}-\lambda_{j}(\omega) z_{j} \xi_{j}=P_{\operatorname{ker}\left(H_{\omega}-\lambda_{j}\right)}\left(\sum_{|m+n|=2} z^{m} \bar{z}^{n} R_{m, n}(\omega)+\sum_{|m+n|=3} z^{m} \bar{z}^{n} R_{m, n}^{(1)}(\omega)\right. \\
& -\sum_{\left|m^{\prime}+n^{\prime}\right|=1} \sum_{|m+n|=2} z^{m+m^{\prime}} \bar{z}^{n+n^{\prime}} A_{m^{\prime}, n^{\prime}}(\omega) R_{H_{\omega_{0}}}^{+}\left((m-n) \cdot \lambda\left(\omega_{0}\right)\right) P_{c}\left(H_{\omega}\right) R_{m, n}(\omega) \\
& +\sum_{|m+n|=1} z^{m} \bar{z}^{n} A_{m, n}(\omega) f_{2}+O\left(f^{2}\right)+O_{l o c}\left(z^{4}\right) .
\end{aligned}
$$

Here recall $P_{\operatorname{ker}\left(H_{\omega}-\lambda_{j}\right)}=s_{j} \xi_{j}\left\langle\quad, \sigma_{3} \xi_{j}\right\rangle$ with $s_{j}=\left\langle\xi_{j}, \sigma_{3} \xi_{j}\right\rangle$ the signature of $\lambda_{j}$, that is either 1 or -1 and with $s_{1}=-1$. By standard normal forms arguments there exists a change of variables $\zeta_{j}=z_{j}+\sum_{|m+n|=2}^{3} p_{j, m, n}(\omega) z^{m} \bar{z}^{n}$ with $\Im p_{j, m, n}=0$ for $|m+n|=2$ such that below we have $\Im a_{j, \ell}(\omega)=0$ and

$$
\begin{aligned}
& i \dot{\zeta}_{j} \xi_{j}-\lambda_{j}(\omega) \zeta_{j} \xi_{j}=\xi_{j} \sum_{\ell} a_{j, \ell}(\omega)\left|\zeta_{\ell}\right|^{2} \zeta_{j}+O_{l o c}\left(\zeta f_{2}\right)+O\left(f^{2}\right)+O_{l o c}\left(\zeta^{4}\right) \\
& -\sum_{\ell}\left|\zeta_{\ell}\right|^{2} \zeta_{j} P_{\operatorname{ker}\left(H_{\omega}-\lambda_{j}\right)} A_{0, \delta_{\ell}}(\omega) R_{H_{\omega_{0}}}^{+}\left(\lambda_{\ell}\left(\omega_{0}\right)+\lambda_{j}\left(\omega_{0}\right)\right) P_{c}\left(H_{\omega}\right) R_{\delta_{\ell}+\delta_{j}, 0}(\omega)
\end{aligned}
$$

where $O_{l o c}\left(\zeta^{n} f_{2}\right)=\sum_{\ell} O_{l o c}\left(\zeta_{\ell}^{n} f_{2}\right)$. Applying $\left\langle, \sigma_{3} \xi_{j}\right\rangle$ to (3.13) and recalling $P_{\operatorname{ker}\left(H_{\omega}-\lambda_{j}\right)}=s_{j} \xi_{j}\left\langle\quad, \sigma_{3} \xi_{j}\right\rangle$ we get

$$
\begin{aligned}
& \left(i \dot{\zeta}_{j}-\lambda_{j}(\omega) \zeta_{j}\right) s_{j}=s_{j} \sum_{\ell} a_{j, \ell}(\omega)\left|\zeta_{\ell}\right|^{2} \zeta_{j}+\left\langle O_{l o c}\left(\zeta f_{2}\right)+O\left(f^{2}\right)+O_{l o c}\left(\zeta^{4}\right), \sigma_{3} \xi_{j}\right\rangle- \\
& -\sum_{\ell}\left|\zeta_{\ell}\right|^{2} \zeta_{j} s_{j}\left\langle\xi_{j}, \sigma_{3} \xi_{j}\right\rangle\left\langle A_{0, \delta_{\ell}}(\omega) R_{H_{\omega_{0}}}^{+}\left(\lambda_{\ell}\left(\omega_{0}\right)+\lambda_{j}\left(\omega_{0}\right)\right) P_{c}\left(H_{\omega}\right) R_{\delta_{\ell}+\delta_{j}, 0}(\omega), \sigma_{3} \xi_{j}\right\rangle .
\end{aligned}
$$

So

$$
\begin{aligned}
& i \dot{\zeta}_{j}-\lambda_{j}(\omega) \zeta_{j}=\sum_{\ell} a_{j, \ell}(\omega)\left|\zeta_{\ell}\right|^{2} \zeta_{j}+\left\langle O_{l o c}\left(\zeta f_{2}\right)+O\left(f^{2}\right)+O_{l o c}\left(\zeta^{4}\right), \sigma_{3} \xi_{j}\right\rangle- \\
& -s_{j} \sum_{\ell}\left|\zeta_{\ell}\right|^{2} \zeta_{j}\left\langle A_{0, \delta_{\ell}}(\omega) R_{H_{\omega_{0}}}^{+}\left(\lambda_{\ell}\left(\omega_{0}\right)+\lambda_{j}\left(\omega_{0}\right)\right) P_{c}\left(H_{\omega}\right) R_{\delta_{\ell}+\delta_{j}, 0}(\omega), \sigma_{3} \xi_{j}\right\rangle .
\end{aligned}
$$


Recall that $\Im a_{j, \ell}(\omega)=0$. Then multiplying $(3.14)$ by $\bar{\zeta}_{j}$ we get

$$
\begin{aligned}
& \frac{1}{2} \frac{d}{d t}\left|\zeta_{j}\right|^{2}=-s_{j} \sum_{\ell}\left(\Gamma_{j, \ell}\left(\omega_{0}\right)+o(1)\right)\left|\zeta_{\ell}\right|^{2}\left|\zeta_{j}\right|^{2}+ \\
& +\Im\left[\overline{\zeta_{j}}\left\langle O_{l o c}\left(\zeta f_{2}\right)+O\left(f^{2}\right)+O_{l o c}\left(\zeta^{4}\right), \sigma_{3} \xi_{j}\right\rangle\right]
\end{aligned}
$$

where we use $\omega-\omega_{0}=O(\epsilon)$,

$$
\Gamma_{j, \ell}(\omega)=\Im\left\langle A_{0, \delta_{\ell}}(\omega) R_{H_{\omega}}^{+}\left(\lambda_{\ell}(\omega)+\lambda_{j}(\omega)\right) P_{c}\left(H_{\omega}\right) R_{\delta_{\ell}+\delta_{j}, 0}(\omega), \sigma_{3} \xi_{j}(\omega)\right\rangle
$$

and the continuous dependence in $\omega$ of $A_{0, \delta_{\ell}}(\omega), R_{\delta_{\ell}+\delta_{j}, 0}(\omega)$ and $P_{c}\left(H_{\omega}\right)$. By Lemma 3.4 we have $\Gamma_{j, \ell}(\omega) \geq 0$. Now we assume the following hypothesis:

Hypothesis 3.7. We suppose that $\Gamma_{j, j}(\omega)>0$ for any $j$.

Remark. Since $2 \lambda_{j}(\omega)>\omega$ for any $j$, Hypothesis 3.7 looks like a generic condition by Lemma 3.4.

By $s_{1}=-1$, integrating (3.15) we get for a fixed $\Gamma>0$ and for $s \gg 1$ fixed

$$
\begin{aligned}
& \left|\zeta_{1}(t)\right|^{2} \geq\left|\zeta_{1}(0)\right|^{2}+\Gamma \int_{0}^{t}\left|\zeta_{1}(\tau)\right|^{4} d \tau-c(\omega)\left(\int_{0}^{t}|\zeta(\tau)|^{4} d \tau\right)^{\frac{1}{2}}\left\|f_{2}\right\|_{L^{2}\left((0, t), L_{x}^{2,-s}\right)} \\
& +o(1)\left(\|f\|_{L^{2}\left((0, t), L_{x}^{2,-s}\right)}^{2}+\int_{0}^{t}|\zeta(\tau)|^{4} d \tau\right) .
\end{aligned}
$$

Similarly, for all the $j$ we have

$$
\begin{aligned}
& \Gamma \int_{0}^{t}\left|\zeta_{j}(\tau)\right|^{4} d \tau \leq\left|\zeta_{j}(t)\right|^{2}+\left|\zeta_{j}(0)\right|^{2}+c(\omega)\left(\int_{0}^{t}|\zeta(\tau)|^{4} d \tau\right)^{\frac{1}{2}}\left\|f_{2}\right\|_{L^{2}\left((0, t), L_{x}^{2,-s}\right)} \\
& +o(1)\left(\|f\|_{L^{2}\left((0, t), L_{x}^{2,-s}\right)}^{2}+\int_{0}^{t}|\zeta(\tau)|^{4} d \tau\right) .
\end{aligned}
$$

For any fixed $C_{1} \gtrsim 1$ there is an $T>0$ such that we have $\left\|\zeta_{j}\right\|_{L^{4}(0, T)}^{2} \leq C_{1} \epsilon$. By Lemma 3.5 we conclude $\|f\|_{L_{t}^{2}\left((0, T), L_{x}^{2,-s}\right)}<C\left(C_{1}\right) \epsilon$ by $\epsilon \geq \delta>0$ for $\epsilon$ sufficiently small. By Lemma 3.6 we have $\left\|f_{2}\right\|_{L_{t}^{2}\left((0, T), L_{x}^{2,-s}\right)}<c_{0} \delta+O\left(\epsilon^{2}\right)$ for a fixed constant $c_{0} \approx 1$. Then by (3.17) and by orbital stability we get $\|\zeta\|_{L^{4}(0, T)}^{2} \leq C_{0} \epsilon$ for some fixed $C_{0}>0$. By a continuity argument, the same argument can be repeated for any $T>0$. Then $\|\zeta\|_{L^{4}(0, \infty)}^{2} \leq C_{0} \epsilon$. This and the fact that $\dot{\zeta} \in L_{t}^{\infty}$ implies $\lim _{t \rightarrow \infty} \zeta(t)=0$. We claim that this is incompatible with (3.16). For $t$ sufficiently large $|\zeta(t)|^{2}<\delta^{2} / 2$. Then by (3.17) we get for a fixed $c$ 


$$
\begin{aligned}
& (\Gamma+o(1)) \int_{0}^{t}|\zeta(s)|^{4} d s \leq c \delta^{2}+c(\omega)\left(\int_{0}^{t}|\zeta(s)|^{4} d s\right)^{\frac{1}{2}}\left\|f_{2}\right\|_{L^{2}\left((0, t), L_{x}^{2,-s}\right)} \\
& +o(1)\|f\|_{L^{2}\left((0, t), L_{x}^{2,-s}\right)}^{2} .
\end{aligned}
$$

If $\Gamma\left(\int_{0}^{t}|\zeta(s)|^{4} d s\right)^{\frac{1}{2}} \geq 2 c(\omega)\left\|f_{2}\right\|_{L^{2}\left((0, t), L_{x}^{2,-s}\right)}$, then by $(3.18)$

$$
\int_{0}^{t}|\zeta(s)|^{4} d s \approx(\Gamma / 2+o(1)) \int_{0}^{t}|\zeta(s)|^{4} d s \leq c \delta^{2}+o(1)\|f\|_{L^{2}\left((0, t), L_{x}^{2,-s}\right)}^{2} .
$$

Then, by the argument in Lemma 3.5 we get $\|f\|_{L^{2}\left((0, t), L_{x}^{2,-s}\right)} \leq c_{1} \delta$ for fixed $c_{1}$ and this implies $\int_{0}^{t}|\zeta(s)|^{4} d s \leq c_{2} \delta^{2}$ for fixed $c_{2}$. If instead for $\varepsilon=\left(\int_{0}^{t}|\zeta(s)|^{4} d s\right)^{\frac{1}{2}}$ we have $\Gamma \varepsilon<2 c(\omega)\left\|f_{2}\right\|_{L^{2}\left((0, t), L_{x}^{2,-s}\right)}$, then by Lemma 3.6 we get $\varepsilon \leq c_{0} \delta+O\left(\delta^{2}+\epsilon(\varepsilon+\right.$ $\delta)$ ). This implies $\varepsilon \leq c_{2} \delta$ for fixed $c_{2}$. So in all cases we get $\|\zeta\|_{L^{4}(0, \infty)}^{2} \leq c_{2} \delta$. We pick now initial conditions $f(0)=0,\left|\zeta_{1}(0)\right|=\delta$ and $\zeta_{j}(0)=0$ for $j>1$. Then, by (3.16) we get

$$
\begin{aligned}
& -\delta^{2} / 2 \geq \Gamma \int_{0}^{t}\left|\zeta_{1}(\tau)\right|^{4} d \tau-c(\omega)\left(\int_{0}^{t}|\zeta(\tau)|^{4} d \tau\right)^{\frac{1}{2}}\left\|f_{2}\right\|_{L^{2}\left((0, t), L_{x}^{2,-s}\right)} \\
& +o(1)\left(\|f\|_{L^{2}\left((0, t), L_{x}^{2,-s}\right)}^{2,-}+\int_{0}^{t}|\zeta(\tau)|^{4} d \tau\right) .
\end{aligned}
$$

By Lemma 3.5 we conclude $\|f\|_{L^{2}\left((0, \infty), L_{x}^{2,-s}\right)}<C\left(c_{1}\right) \delta$. Since $f(0)=0$, by Lemma 3.6 we have $\left\|f_{2}\right\|_{L^{2}\left((0, \infty), L_{x}^{2,-s}\right)}<C \epsilon \delta$. So we have

$$
-\delta^{2} / 2>\Gamma\left\|\zeta_{1}\right\|_{L^{4}(0, \infty)}^{4}+O\left(o(1) \delta^{2}\right) \geq O\left(o(1) \delta^{2}\right) .
$$

By $\left|O\left(o(1) \delta^{2}\right)\right| \ll \delta$ it follows that (3.19) is absurd. But (3.19) is a consequence of the fact that we are assuming the orbital stability of $e^{i t \omega_{0}} \phi_{\omega_{0}}$. This implies that $e^{i t \omega_{0}} \phi_{\omega_{0}}$ is orbitally unstable. Theorem 3.2 is proved.

\section{$\S 4$ The CASE WHEn THE INTERNAL MODES ARE NOT NECESSARILY CLOSE TO THE CONTINUOUS SPECTRUM}

In this section we we consider the following two hypotheses:

(H8') $H_{\omega}$ has a certain number of simple positive eigenvalues with $0<N_{j} \lambda_{j}(\omega)<\omega<$ $\left(N_{j}+1\right) \lambda_{j}(\omega)$ with $N_{j}$ integers with $N_{j} \geq 1$. $H_{\omega}$ does not have other eigenvalues and $\pm \omega$ are not resonances. We set $N=\max _{j} N_{j}$. 
(H9') For multi indexes $m=\left(m_{1}, m_{2}, \ldots\right)$ and $n=\left(n_{1}, \ldots\right)$, setting $\lambda(\omega)=\left(\lambda_{1}(\omega), \ldots\right)$ and $(m-n) \cdot \lambda=\sum\left(m_{j}-n_{j}\right) \lambda_{j}$, we have the following two non resonance hypotheses:

(i) $\quad(m-n) \cdot \lambda(\omega)=0$ implies $m=n$ if $|m| \leq N+2$ and $|n| \leq N+2$;

(ii) $(m-n) \cdot \lambda(\omega) \neq \omega$ for all $(m, n)$ with $|m|+|n| \leq N+2$.

(H10') We assume the non degeneracy Hypotheses 4.4 and 4.5.

$\left(\mathrm{H} 11^{\prime}\right) \quad \beta(t) \in C^{N+2}(\mathbb{R}, \mathbb{R})$.

Under hypothesis (H8'), if $\xi$ is a generator of $\operatorname{ker}\left(H_{\omega}-\lambda_{j}\right)$ for any $j$, then $\left\langle\xi, \sigma_{3} \xi\right\rangle \neq 0$.

Theorem 4.1. Under hypotheses (H1-7) and (H8'-11') the excited states $e^{i \omega t} \phi_{\omega}(x)$ are orbitally unstable.

The structure of the proof is similar to Theorem 3.2, only more complicate because we perform the normal form argument in $[\mathrm{CM}]$. Given two vectors we will write $\vec{a} \leq \vec{b}$ if $a_{j} \leq b_{j}$ for all components. If this happens we write $\vec{a}<\vec{b}$ if we have $a_{j}<b_{j}$ for at least one $j$. We will set $(m-n) \cdot \lambda=\sum_{j}(m-n)_{j} \lambda_{j}$. We will say that $m \in$ Res if:

(i) $m=\left(m_{1}, m_{2}, \ldots\right)$ with $m_{j} \in \mathbb{N} \cup\{0\}$ for all $j$;

(ii) $m \cdot \lambda(\omega)>\omega$;

(iii) Given an $n$ satisfying (i) and with $n<m$ with $m \in R e s$, then $n \cdot \lambda(\omega)<\omega$.

We assume by absurd that the excited state $e^{i \omega_{0} t} \phi_{\omega_{0}}(x)$ is orbitally stable. We rewrite formula (3.4) and we consider the expansion

$$
\sigma_{3} \sigma_{1} G^{\prime}(R)=\sum_{|m+n|=2}^{2 N+1} R_{m, n}(\omega) z^{m} \bar{z}^{n}+\sum_{|m+n|=1}^{N} z^{m} \bar{z}^{n} A_{m, n}(\omega) f+O\left(f^{2}\right)+\cdots .
$$

$A_{m, n}(\omega)$ and $R_{m, n}(\omega)$ are real with $\sigma_{1} R_{m, n}=-R_{n, m}$ and $A_{m, n}=-\sigma_{1} A_{n, m} \sigma_{1}$. We express (3.4) as

$$
\begin{aligned}
& i f_{t}=\left(H_{\omega(t)}+P_{c}\left(H_{\omega}\right) \sigma_{3} \dot{\gamma}\right) f+\sum_{|m+n|=2}^{N+1} z^{m} \bar{z}^{n} P_{c}\left(H_{\omega}\right) R_{m, n}^{(1)}(\omega) \\
& +\sum_{|m+n|=1}^{N} z^{m} \bar{z}^{n} P_{c}\left(H_{\omega}\right) A_{m, n}^{(1)}(\omega) f+O\left(f^{2}\right)+\sum_{m \in \operatorname{Res}} O_{l o c}\left(\left|z^{m}\right|\right),
\end{aligned}
$$

and for $\widetilde{R}_{m, n}^{(1)}=R_{m, n}^{(1)}$

$$
\begin{aligned}
i \dot{z}_{j} \xi_{j}-\lambda_{j}(\omega) z_{j} \xi_{j} & =P_{\operatorname{ker}\left(H_{\omega}-\lambda_{j}\right)}\left(\sum_{|m+n|=2}^{2 N+1} z^{m} \bar{z}^{n} \widetilde{R}_{m, n}^{(1)}(\omega)\right. \\
& \left.+\sum_{|m+n|=1}^{N} z^{m} \bar{z}^{n} A_{m, n}^{(1)}(\omega) f+O\left(f^{2}\right)+\sum_{m \in \text { Res }} O_{l o c}\left(\left|z^{m}\right|\right)\right) .
\end{aligned}
$$


We consider $k=1,2, \ldots N$ and set $f=f_{k}$ and $z_{(k), j}=z_{j}$ for $k=1$. The other $f_{k}$ and $z_{(k), j}$ are defined below by induction. In (4.1) for $\ell=1$ we have

$$
\sigma_{1} f_{\ell}=\bar{f}_{\ell}, A_{m, n}^{(\ell)}, R_{m, n}^{(\ell)} \text { and } \widetilde{R}_{m, n}^{(\ell)} \text { are real, }
$$

exponentially decreasing in $x$ and $C^{1}$ in $(\omega, x) ; \sigma_{1} R_{m, n}^{(\ell)}=-R_{n, m}^{(\ell)}$.

In the ODE's there will be error terms of the form

$$
E_{O D E}(k)=\sum_{M \in R e s}\left\{O\left(\left|z_{(k)}^{M}\right|^{2}\right)+O\left(z_{(k)}^{M} f_{k}\right)\right\}+O\left(f_{k}^{2}\right)+O\left(\beta\left(\left|f_{k}\right|^{2} f_{k}\right)\right) \text {. }
$$

In the PDE's there will be error terms of the form

$$
E_{P D E}(k)=\sum_{M \in \operatorname{Res}} O_{l o c}\left(\left|z_{(k)}\right|^{M} \mid\right)\left|z_{(k)}\right|+O_{l o c}\left(z_{(k)} f_{k}\right)+O\left(f_{k}^{2}\right)+O\left(\beta\left(\left|f_{k}\right|^{2} f_{k}\right)\right) .
$$

Then we define $f_{1}=f$ and, summing only over $(m, n)$ with $|(m-n) \cdot \lambda|<\omega$,

$$
f_{k}=f_{k-1}+\sum_{|m+n|=k} R_{H_{\omega}}((m-n) \cdot \lambda) P_{c}\left(H_{\omega}\right) R_{m, n}^{(k-1)}(\omega) z_{(k-1)}^{m} \bar{z}_{(k-1)}^{n} .
$$

By $\sigma_{1} R_{m, n}^{(k-1)}=-R_{n, m}^{(k-1)}$, by the fact that $R_{m, n}^{(k-1)}$ is real and by $\sigma_{1} H_{\omega}=-H_{\omega} \sigma_{1}$ we get $\sigma_{1} f_{k}=\bar{f}_{k}$. Starting from $\widetilde{R}_{m, n}^{(1)}=R_{m, n}^{(1)}$ and summing only over $(m, n)$ with $\lambda_{j}(\omega) \neq(m-n) \cdot \lambda(\omega)$, we set

$$
z_{(k), j} \xi_{j}=z_{(k-1), j} \xi_{j}+\sum_{|m+n|=k} P_{\operatorname{ker}\left(H_{\omega}-\lambda_{j}\right)} \widetilde{R}_{m, n}^{(k-1)}(\omega) \frac{z_{(k-1)}^{m} \bar{z}_{(k-1)}^{n}}{\lambda_{j}-(m-n) \cdot \lambda} .
$$

We get the equations

$$
\begin{aligned}
& i \partial_{t} f_{k}=\left(H_{\omega}+\sigma_{3} \dot{\gamma}\right) f_{k}+E_{P D E}(k)+ \\
& \left.\quad \sum_{k+1 \leq|m+n| \leq N+1}^{(k)} R_{m, n}^{(k)}(\omega) z_{(k)}^{m} \bar{z}_{(k)}^{n} \text { (sum over pairs with }|(m-n) \cdot \lambda|<\omega\right) \\
& \left.+\sum_{2 \leq|m+n| \leq N+1} R_{m, n}^{(k)}(\omega) z_{(k)}^{m} \bar{z}_{(k)}^{n} \text { (sum over pairs with }|(m-n) \cdot \lambda|>\omega\right) ; \\
& \quad i \dot{z}_{(k), j} \xi_{j}-\lambda_{j}(\omega) z_{(k), j} \xi_{j}=\xi_{j} \sum_{|m|=1}^{N} a_{j, m}^{(k)}(\omega)\left|z_{(k)}^{m}\right|^{2} z_{(k), j}+E_{O D E}(k)+ \\
& \quad \sum_{|m+n|=k+1}^{N+1} P_{\operatorname{ker}\left(H_{\omega}-\lambda_{j}\right)} \widetilde{R}_{m, n}^{(k)}(\omega) z_{(k)}^{m} \bar{z}_{(k)}^{n}\left(\text { with }(m-n) \cdot \lambda \neq \lambda_{j}\right) \\
& +\sum_{|m+n|=1}^{N} z_{(k)}^{m} \bar{z}_{(k)}^{n} P_{\operatorname{ker}\left(H_{\omega}-\lambda_{j}\right)} A_{m, n}^{(k)}(\omega) f_{k} .
\end{aligned}
$$


The coefficients in $\left(4.4_{k}\right)$ are real because their entries are products of entries of the coefficients in (4.3), which are real, with Taylor coefficients of the rhs in $\left(4.4_{k-1}\right)$ at $z_{(k-1)}=\bar{z}_{(k-1)}=0$ and $f_{k-1}=0$, which are also real. So in particular $\Im\left[a_{j, m}^{(k)}(\omega)\right]=$ 0 . Since by (4.3) and by induction we have $\sigma_{1} f_{k}=\bar{f}_{k}$, taking complex conjugate in the $f_{k}$ equation in $\left(4.4_{k}\right)$ we get $\sigma_{1} R_{m, n}^{(k)}=-R_{n, m}^{(k)}$. At the step $k=N$, we can define

$$
\zeta_{j}=z_{(N), j}+p_{j}\left(z_{(N)}, \bar{z}_{(N)}\right)+\sum_{1 \leq|m+n| \leq N} z_{(N)}^{m} \bar{z}_{(N)}^{n}\left\langle f_{N}, \alpha_{j m n}\right\rangle, \text { with: }
$$

$\alpha_{j m n}$ vectors with entries which are real valued exponentially decreasing functions; $p_{j}$ polynomials in $\left(z_{(N)}, \bar{z}_{(N)}\right)$ with real coefficients and whose monomials have degree not smaller than $N+1$. The above transformation can be chosen so that:

$$
\begin{aligned}
& i \dot{\zeta}_{j} \xi_{j}-\lambda_{j}(\omega) \zeta_{j} \xi_{j}=\xi_{j} \sum_{1 \leq|m| \leq N} b_{j, m}(\omega)\left|\zeta^{m}\right|^{2} \zeta+E_{O D E}+ \\
& +\sum_{n+\delta_{j} \in \operatorname{Res}} \bar{\zeta}^{n} P_{\operatorname{ker}\left(H_{\omega}-\lambda_{j}\right)} A_{0, n}^{(N)}(\omega) f_{N}
\end{aligned}
$$

with $b_{j, m}(\omega)$ real and $E_{O D E}$ an error term

$$
E_{O D E}=\sum_{M \in \text { Res }}\left\{O\left(\left|\zeta^{M}\right|^{2}\right)+O\left(\zeta^{M} f_{N}\right)\right\}+O\left(f_{N}^{2}\right)+O\left(\beta\left(\left|f_{N}\right|^{2} f_{N}\right)\right) .
$$

We write

$$
\begin{aligned}
& i \partial_{t} P_{c}\left(\omega_{0}\right) f_{N}=\left\{H_{\omega_{0}}+\left(\dot{\gamma}+\omega-\omega_{0}\right)\left(P_{+}\left(\omega_{0}\right)-P_{-}\left(\omega_{0}\right)\right)\right\} P_{c}\left(\omega_{0}\right) f_{N}+ \\
& +P_{c}\left(\omega_{0}\right) \widetilde{E}_{P D E}(N)+\sum_{2 \leq|m+n| \leq N+1} P_{c}\left(\omega_{0}\right) R_{m, n}^{(N)}\left(\omega_{0}\right) \zeta^{m} \bar{\zeta}^{n}
\end{aligned}
$$

where $|(m-n) \cdot \lambda|>\omega$ for $|m+n| \leq N$ and with

$$
\begin{aligned}
& \widetilde{E}_{P D E}(N)=E_{P D E}(N)+\sum_{2 \leq|m+n| \leq N+1} P_{c}\left(\omega_{0}\right)\left(R_{m, n}^{(N)}(\omega)-R_{m, n}^{(N)}\left(\omega_{0}\right)\right) \zeta^{m} \bar{\zeta}^{n}+ \\
& +\left(\dot{\gamma}+\omega-\omega_{0}\right)\left(P_{c}\left(\omega_{0}\right) \sigma_{3}-\left(P_{+}\left(\omega_{0}\right)-P_{-}\left(\omega_{0}\right)\right)\right) f_{N}+\left(V(\omega)-V\left(\omega_{0}\right)\right) f_{N} \\
& +\left(\dot{\gamma}+\omega-\omega_{0}\right)\left(P_{c}(\omega)-P_{c}\left(\omega_{0}\right)\right) \sigma_{3} f_{N} .
\end{aligned}
$$

Then proof of the following is almost the same of Lemma 3.5: 
Lemma 4.2. Let $0<\delta<\epsilon$ be as in the Definition 3.1 of linear stability. For any $C_{1}>0$ there are a $\varepsilon\left(C_{1}\right)>0$ and a $C\left(C_{1}\right)$ such that if, for $0<\varepsilon<\varepsilon\left(C_{1}\right)$, we have $\sum_{m \in \text { Res }}\left\|\zeta^{m}\right\|_{L_{t}^{2}(0, T)} \leq C_{1} \epsilon$ for all $j$, then for all admissible pairs $(p, q)$ we have for a fixed $c_{0}$

$$
\left\|f_{N}\right\|_{L_{t}^{p}\left((0, T), W_{x}^{1, q}\right)}<c_{0} \delta+C\left(C_{1}\right) \varepsilon .
$$

For $\left|(m-n) \cdot \lambda\left(\omega_{0}\right)\right|>\omega_{0}$ if $|m+n| \leq N$ in the sum (4.6) below, we set

$$
f_{N}=-\sum_{2 \leq|m+n| \leq N+1} R_{H_{\omega_{0}}}^{+}\left((m-n) \cdot \lambda\left(\omega_{0}\right)\right) P_{c}(\omega) R_{m, n}^{(N)}\left(\omega_{0}\right) \zeta^{m} \bar{\zeta}^{n}+f_{N+1} .
$$

Then we get

$$
\begin{gathered}
i \partial_{t} P_{c}\left(\omega_{0}\right) f_{N+1}=\left(H_{\omega_{0}}+\left(\dot{\gamma}+\omega-\omega_{0}\right)\left(P_{+}\left(\omega_{0}\right)-P_{-}\left(\omega_{0}\right)\right)\right) P_{c}\left(\omega_{0}\right) f_{N+1}+ \\
\sum O\left(|\zeta|^{|m+n|+1}\right) R_{H_{\omega_{0}}}^{+}\left((m-n) \cdot \lambda\left(\omega_{0}\right)\right) R_{m, n}^{(N)}\left(\omega_{0}\right)+P_{c}\left(\omega_{0}\right) \widetilde{E}_{P D E}(N) .
\end{gathered}
$$

We have $O\left(|\zeta|^{|m+n|+1}\right)=O\left(\left|\zeta^{M} \zeta\right|\right)$ for $M \in$ Res for each factor in (4.7). By a proof similar to Lemma 3.6, see also [CM], we have:

Lemma 4.3. Assume the hypotheses of Lemma 4.2. Then for $s>1$ sufficiently large we can decompose $f_{N+1}=h_{1}+h_{2}+h_{3}+h_{4}$ with:

(1) for a fixed $c_{0}\left(\omega_{0}\right),\left\|h_{1}\right\|_{L_{t}^{2}\left(\mathbb{R}, L_{x}^{2,-s}\right)} \leq c_{0}\left(\omega_{0}\right)\|f(0)\|_{H^{1}} \leq c_{0}\left(\omega_{0}\right) \delta$;

(2) for a fixed $c_{1}\left(\omega_{0}\right),\left\|h_{2}\right\|_{L_{t}^{2}\left(\mathbb{R}, L_{x}^{2,-s}\right)} \leq c_{1}\left(\omega_{0}\right)|z(0)|^{2} \leq c_{1}\left(\omega_{0}\right) \delta^{2}$;

(3) $\left\|h_{3}\right\|_{L_{t}^{2}\left((0, T), L_{x}^{2,-s}\right)} \leq O(\epsilon(\varepsilon+\delta))$;

(4) for all admissible pairs $\left\|h_{4}\right\|_{L_{t}^{r}\left((0, T), L_{x}^{p}\right)}=O(\epsilon \varepsilon)$.

Substituting $f_{N}$ in (4.5) with the right hand side of (4.6) we get

$$
\begin{aligned}
& i \dot{\zeta}_{j} \xi_{j}-\lambda_{j}(\omega) \zeta_{j} \xi_{j}=\xi_{j} \sum_{1 \leq|m| \leq N} b_{j, m}(\omega)\left|\zeta^{m}\right|^{2} \zeta+\sum_{2 \leq|m+n| \leq N+1} \sum_{n+\delta_{j} \in \operatorname{Res}} \zeta^{m} \bar{\zeta}^{\widetilde{n}+n} \times \\
& P_{\operatorname{ker}\left(H_{\omega}-\lambda_{j}\right)} A_{0, n}^{(N)}(\omega) R_{H_{\omega_{0}}}^{+}\left((m-n) \cdot \lambda\left(\omega_{0}\right)\right) P_{c}(\omega) R_{m, n}^{(N)}\left(\omega_{0}\right) \\
& +\sum_{n+\delta_{j} \in \operatorname{Res}} \bar{\zeta}^{n} P_{\operatorname{ker}\left(H_{\omega}-\lambda_{j}\right)} A_{0, n}^{(N)}(\omega) f_{N+1}+E_{O D E}
\end{aligned}
$$

where $|(m-n) \cdot \lambda|>\omega$ for $|m+n| \leq N$ in the above formula. We considerate new change of variables $\widetilde{\zeta}_{j}=\zeta_{j}+p_{j}(\zeta, \bar{\zeta})$ such that

$$
\begin{aligned}
& \left(i \dot{\widetilde{\zeta}}_{j}-\lambda_{j}(\omega) \widetilde{\zeta}_{j}\right) \xi_{j}=\xi_{j} \sum_{1 \leq|m| \leq N} \widetilde{a}_{j, m}(\omega)\left|\widetilde{\zeta}^{m}\right|^{2} \widetilde{\zeta}_{j}+E_{O D E}(N)- \\
& \quad \sum_{m+\delta_{j} \in \operatorname{Res}}\left|\widetilde{\zeta}^{m}\right|^{2} \widetilde{\zeta}_{j} P_{\operatorname{ker}\left(H_{\omega}-\lambda_{j}\right)} A_{0, m}^{(N)}(\omega) R_{H_{\omega_{0}}}^{+}\left(m \cdot \lambda\left(\omega_{0}\right)+\lambda_{j}\left(\omega_{0}\right)\right) R_{m+\delta_{j}, 0}^{(N)}\left(\omega_{0}\right) \\
& -\sum_{m+\delta_{j} \in \operatorname{Res}} \stackrel{\widetilde{\zeta}}{m}^{m} P_{\operatorname{ker}\left(H_{\omega}-\lambda_{j}\right)} A_{0, m}^{(N)}(\omega) f_{N+1}
\end{aligned}
$$


with $\widetilde{a}_{j, m}, A_{0, m}^{(N)}$ and $R_{m+\delta_{j}, 0}^{(N)}$ real and with all the $m$ such that $m+\delta_{j} \in R e s$. It is possible to choose $p_{j}(\omega, z, \bar{z})$ as polynomials with monomials $z^{m} \bar{z}^{n+\widetilde{n}}$ which, by $(m+n) \cdot \lambda>\omega$, are $O\left(z^{M}\right)$ for $M \in$ Res. This implies $\sum_{M \in R e s}\left\|\zeta^{M}(t)\right\|_{L_{t}^{2}} \approx$ $\sum_{M \in \operatorname{Res}}\left\|\widetilde{\zeta}^{M}(t)\right\|_{L_{t}^{2}}$.

Applying $\left\langle, \sigma_{3} \xi_{j}\right\rangle$ to $(4.8)$ and recalling $P_{\operatorname{ker}\left(H_{\omega}-\lambda_{j}\right)}=s_{j} \xi_{j}\left\langle\quad, \sigma_{3} \xi_{j}\right\rangle$ we get

$$
\begin{aligned}
& i \dot{\widetilde{\zeta}}_{j}-\lambda_{j}(\omega) \widetilde{\zeta}_{j}=\sum_{1 \leq|m| \leq N} \widetilde{a}_{j, m}(\omega)\left|\widetilde{\zeta}^{m}\right|^{2} \widetilde{\zeta}_{j}+\left\langle E_{O D E}(N) \sigma_{3} \xi_{j}\right\rangle-s_{j} \times \\
& \sum_{m+\delta_{j} \in \operatorname{Res}}\left|\widetilde{\zeta}^{m}\right|^{2} \widetilde{\zeta}_{j}\left\langle A_{0, m}^{(N)}(\omega) R_{H_{\omega_{0}}}^{+}\left(m \cdot \lambda\left(\omega_{0}\right)+\lambda_{j}\left(\omega_{0}\right)\right) R_{m+\delta_{j}, 0}^{(N)}\left(\omega_{0}\right), \sigma_{3} \xi_{j}\right\rangle \\
& -s_{j} \sum_{m+\delta_{j} \in \operatorname{Res}}\left|\widetilde{\zeta}^{m}\right|^{2} \widetilde{\zeta}_{j}\left\langle A_{0, m}^{(N)}(\omega) f_{N+1}, \sigma_{3} \xi_{j}\right\rangle .
\end{aligned}
$$

We can denote by $\Gamma_{m+\delta_{j}, j}\left(\omega, \omega_{0}\right)$ the quantity $\Gamma_{m+\delta_{j}, j}\left(\omega, \omega_{0}\right)=$

$$
\begin{aligned}
& \Im\left(\left\langle A_{0, m}^{(N)}(\omega) R_{H_{\omega_{0}}^{+}}^{+}\left(m \cdot \lambda\left(\omega_{0}\right)+\lambda_{j}\left(\omega_{0}\right)\right) R_{m+\delta_{j}, 0}^{(N)}\left(\omega_{0}\right), \sigma_{3} \xi_{j}(\omega)\right\rangle\right) \\
& =\pi\left\langle A_{0, m}^{(N)}(\omega) \delta\left(H_{\omega_{0}}-m \cdot \lambda\left(\omega_{0}\right)-\lambda_{j}\left(\omega_{0}\right)\right) P_{c}\left(\omega_{0}\right) R_{m+\delta_{j}, 0}^{(N)}\left(\omega_{0}\right), \sigma_{3} \xi_{j}(\omega)\right\rangle,
\end{aligned}
$$

by $\frac{1}{x-i 0}=P V \frac{1}{x}+i \pi \delta_{0}(x),[\mathrm{Cu} 2]$ and Lemma $4.1[\mathrm{Cu} 3]$ and which can be proved as in Lemma 3.4. We formulate the following hypothesis, which is a conjecture:

Hypothesis 4.4. We have the identity $\Gamma_{m+\delta_{j}, j}(\omega, \omega)=$

$$
=\left(m_{j}+1\right)\left\langle\delta\left(H_{\omega}-m \cdot \lambda(\omega)-\lambda_{j}(\omega)\right) P_{c}(\omega) R_{m+\delta_{j}, 0}^{(N)}(\omega), \sigma_{3} R_{m+\delta_{j}, 0}^{(N)}(\omega)\right\rangle .
$$

If Hypothesis 4.4 holds, then proceeding as in Lemma 3.4 we get $\Gamma_{m+\delta_{j}, j}(\omega, \omega) \geq$ 0 . We then assume the following hypothesis:

Hypothesis 4.5 (non degeneracy hypothesis). We have $\Gamma_{m+\delta_{j}, j}(\omega, \omega)>0$ for any $j$ and any $m+\delta_{j} \in$ Res.

Recall that $\Im a_{j, m}(\omega)=0$. Then by (4.10) we get

$$
\begin{aligned}
& \frac{d}{d t} \frac{\left|\widetilde{\zeta}_{j}\right|^{2}}{2}=-s_{j} \sum_{m+\delta_{j} \in \operatorname{Res}} \Gamma_{m+\delta_{j}, j}\left(\omega, \omega_{0}\right)\left|\widetilde{\zeta}^{m} \widetilde{\zeta}_{j}\right|^{2}+\Im\left(\left\langle E_{O D E}(N), \sigma_{3} \xi_{j}(\omega)\right\rangle \overline{\widetilde{\zeta}}_{j}\right) \\
& +s_{j} \sum_{m+\delta_{j} \in \operatorname{Res}} \Im\left(\left\langle A_{0, m}^{(N)}(\omega) f_{N+1}, \sigma_{3} \xi_{j}(\omega)\right\rangle \overline{\widetilde{\zeta}}^{m} \overline{\widetilde{\zeta}}_{j}\right) .
\end{aligned}
$$


By $s_{1}=-1$ we get for a fixed $\Gamma>0$

$$
\left|\widetilde{\zeta}_{1}(t)\right|^{2} \geq\left|\widetilde{\zeta}_{1}(0)\right|^{2}+\Gamma \int_{0}^{t}\left|\widetilde{\zeta}_{1}(\tau)\right|^{2 N_{1}+2} d \tau+o(1)\left(\left\|f_{N+1}\right\|_{L_{t}^{2} L_{x}^{2,-s}}^{2}+\sum_{m \in \operatorname{Res}}\left\|\widetilde{\zeta}^{m}\right\|_{L_{t}^{2}}^{2}\right)
$$

and for all the $m \in$ Res

$$
\begin{aligned}
& \Gamma \int_{0}^{t}\left|\widetilde{\zeta}^{m}(\tau)\right|^{2} d \tau \leq \sum_{j}\left|\zeta_{j}(t)\right|^{2} \\
& +\sum_{j}\left|\widetilde{\zeta}_{j}(0)\right|^{2}+o(1)\left(\left\|f_{N+1}\right\|_{L_{t}^{2} L_{x}^{2,-s}}^{2}+\sum_{m \in R e s}\left\|\widetilde{\zeta}^{m}\right\|_{L_{t}^{2}}^{2}\right) .
\end{aligned}
$$

By the same argument in $\S 3$ we conclude for a fixed $C_{1}$

$$
\sum_{m \in \operatorname{Res}}\left\|\widetilde{\zeta}^{m}\right\|_{L^{2}(0, \infty)}^{2}<C_{1} \epsilon
$$

Then $\lim _{t \rightarrow \infty} \widetilde{\zeta}_{j}(t)=0$. We claim that this is incompatible with (4.11). For $t$ large $|\widetilde{\zeta}(t)|^{2}<\delta^{2} / 2$. Then by (4.12) we get for a fixed $c$

$$
\begin{aligned}
& (\Gamma+o(1)) \sum_{m \in \operatorname{Res}} \int_{0}^{t}\left|\widetilde{\zeta}^{m}(\tau)\right|^{2} d \tau \leq c \delta^{2}+ \\
& +c(\omega)\left(\sum_{m \in \operatorname{Res}} \int_{0}^{t}\left|\widetilde{\zeta}^{m}(\tau)\right|^{2} d \tau\right)^{\frac{1}{2}}\left\|f_{N+1}\right\|_{L^{2}\left((0, t), L_{x}^{2,-s}\right)}+o(1)\left\|f_{N}\right\|_{L^{2}\left((0, t), L_{x}^{2,-s}\right)}^{2} .
\end{aligned}
$$

As in $\S 3$ this yields $\sum_{m \in R e s}\left\|\widetilde{\zeta}^{m}\right\|_{L^{2}(0, \infty)}^{2} \leq c_{2} \delta$ for fixed $c_{2}$. We pick initial conditions $f_{N}(0)=0,\left|\widetilde{\zeta}_{1}(0)\right|=\delta$ and $\widetilde{\zeta}_{j}(0)=0$ for $j>1$. Then by (4.11) we get

$$
\begin{aligned}
& -\delta^{2} / 2 \geq \Gamma \int_{0}^{t}\left|\widetilde{\zeta}_{1}(\tau)\right|^{2 N_{1}+2} d \tau \\
& -c(\omega)\left(\sum_{m \in R e s} \int_{0}^{t}\left|\widetilde{\zeta}^{m}(\tau)\right|^{2} d \tau\right)^{\frac{1}{2}}\left\|f_{N+1}\right\|_{L^{2}\left((0, t), L_{x}^{2,-s}\right)} \\
& +o(1)\left(\left\|f_{N}\right\|_{L^{2}\left((0, t), L_{x}^{2,-s}\right)}^{2}+\sum_{m \in \operatorname{Res}} \int_{0}^{t}\left|\widetilde{\zeta}^{m}(\tau)\right|^{2} d \tau\right) .
\end{aligned}
$$

By Lemma 4.2 we conclude $\left\|f_{N}\right\|_{L^{2}\left((0, \infty), L_{x}^{2,-s}\right)}<C\left(c_{1}\right) \delta$. Since $f_{N}(0)=0$, by Lemma 4.3 we have $\left\|f_{N+1}\right\|_{L^{2}\left((0, \infty), L_{x}^{2,-s}\right)}<C \epsilon \delta$. So as in $\S 3$ we have

$$
-\delta^{2} / 2>\Gamma\left\|\widetilde{\zeta}_{1}\right\|_{L^{2 N_{1}+2}(0, \infty)}^{N_{1}+1}+O\left(o(1) \delta^{2}\right) \geq O\left(o(1) \delta^{2}\right)
$$

which is absurd. So Theorem 4.1 is proved. 


\section{$\S 5$ Completion of Proof of inequality (2.4)}

In this section we assume that $\omega$ is either exceptional of second or third type. We consider a factorization $V=B^{*} A$ of $H_{\omega}=\sigma_{3}(-\Delta+\omega)+V$, with $A, B$ in $C^{2}$, with real entries and exponentially decreasing. As in $\S 2$ we e consider a real matrix $U_{1}(x) \in C_{0}^{\infty}$ and we consider the perturbation $H_{\omega, \epsilon}=H_{\omega}+\epsilon U_{1}$. We also consider a factorization $U_{1}(x)=B_{1}^{*}(x) A(x)$ with $B_{1}^{*}(x)$ with real entries and in $C_{0}^{2}$. We will consider $z \notin[\omega,+\infty)$ with $\Re z>0$ and in some fixed small neighborhood of $\omega$. This $z$ can be expressed as $z=\omega-\zeta^{2}$ with $\Re \zeta>0$ close to 0 . Then we the write $R_{0}(\zeta):=R_{\sigma_{3}(-\Delta+\omega)}(z)$ with integral kernel

$$
R_{0}(x, y, \zeta):=\frac{\sigma_{3}}{4 \pi|x-y|}\left[\begin{array}{cc}
e^{-\zeta|x-y|} & 0 \\
0 & e^{-\sqrt{2 \omega-\zeta^{2}}|x-y|}
\end{array}\right] .
$$

Notice that (5.1) can be continued analytically in $\Re \zeta<0$, but that this continuation does not represent the resolvent $R_{\sigma_{3}(-\Delta+\omega)}\left(\omega-\zeta^{2}\right)$. A Taylor expansion at $\zeta=0$ of (5.1) yields

$$
R_{0}(x, y, \zeta)=\frac{\sigma_{3}}{4 \pi|x-y|}\left[\begin{array}{cc}
1 & 0 \\
0 & e^{-\sqrt{2 \omega}|x-y|}
\end{array}\right]-\left[\begin{array}{cc}
\frac{1}{4 \pi} & 0 \\
0 & 0
\end{array}\right] \zeta+O\left(\zeta^{2}\right) .
$$

We consider the corresponding expansion of $R_{0}(\zeta) \in \mathcal{B}\left(H_{s}^{-1}, H_{-s}^{1}\right), s>5 / 2$,

$$
R_{0}(\zeta)=R_{0}(0)-\zeta G_{1}+O\left(\zeta^{2}\right) .
$$

We set $Q(z)=A R_{H_{\omega}}(z) B_{1}^{*}$ and $Q_{1, \epsilon}(z)=A R_{H_{\omega, \epsilon}}(z) B_{1}^{*}=(1+\epsilon Q(z))^{-1} Q(z)$. Recall that $\mathcal{V}$ was the vector space formed by eigenvalues and resonant vectors of $H_{\omega}$ at $\omega$. In $[\mathrm{CP}]$, following $[\mathrm{JK}]$, it is proved:

Lemma 5.1. There is an $\left(1+A R_{0}(0) B^{*}\right)$ invariant splitting

$$
L^{2}=\operatorname{ker}\left(1+A R_{0}(0) B^{*}\right) \oplus \operatorname{ker}^{\perp}\left(1+B R_{0}(0) A^{*}\right) .
$$

Let $s>1 / 2$. Then $\mathcal{V}=\operatorname{ker}\left(1+R_{0}(0) B^{*} A\right)$. The map $\psi \rightarrow \Psi=-A \psi$ is an isomorphism

$$
\operatorname{ker}\left(1+R_{0}(0) B^{*} A\right) \subset L_{-s}^{2} \rightarrow \operatorname{ker}\left(1+A R_{0}(0) B^{*}\right) \subset L^{2} .
$$

The inverse map is $\Psi \rightarrow R_{0}(0) B^{*} \Psi$.

We will denote by $P \oplus Q$ the projections associated to (5.2).

We will suppose now that (2.4) is not true. This implies that

$$
\inf \left\{\left\langle\sigma_{3} H_{\omega} u, u\right\rangle:\|u\|_{2}=1, u \in H^{1} \cap L_{c}^{2}\left(H_{\omega}\right)\right\}=-\lambda<0 .
$$

In particular, there exists $u \in L_{c}^{2}\left(H_{\omega}\right)$ unitary with $\sigma_{3} H_{\omega} u=-\lambda u$. Then, by standard theory $u \in C^{2}$ with $|u(x)| \lesssim e^{-\sqrt{\omega}|x|}$.

We distinguish now between the cases when $\omega$ is of second and of third type. 


\section{$\S 5.1 \omega$ EXCEPTIONAL OF SECOND TYPE}

We assume here that $\omega$ is an eigenvalue but not a resonance. Let $P_{0}$ be the natural spectral projection in $L^{2}$ on $\mathcal{V}:=\operatorname{ker} H_{\omega}$. By Corollary $4.4[\mathrm{CP}]$ for $s>5 / 2$ and for $\zeta$ near 0 we have in $B\left(H_{s}^{-1}, H_{-s}^{1}\right)$ the expansion

$$
R_{H_{\omega}}\left(\omega-\zeta^{2}\right)=\zeta^{-2} P_{0}-\zeta^{-1} P_{0} V G_{3} V P_{0}+O(1)
$$

with $G_{3}(x, y)=\frac{1}{24 \pi} \operatorname{diag}(1,0)|x-y|^{2}$, i.e. the diagonal $2 \mathrm{x} 2$ matrix with $(1,0)$ on the diagonal. We can write

$$
Q\left(\omega-\zeta^{2}\right)=\zeta^{-2} A P_{0} B_{1}^{*}-\zeta^{-1} A P_{0} V G_{3} V P_{0} B_{1}^{*}+Q_{c}\left(\omega-\zeta^{2}\right)
$$

where $Q_{c}\left(\omega-\zeta^{2}\right)$ admits an analytic extension for $\zeta$ around 0 . We write

$$
\begin{aligned}
& Q_{1, \epsilon}(z)=\left[1+\epsilon\left(1+\epsilon Q_{c}(z)\right)^{-1} \zeta^{-2} K(\epsilon, \zeta)\right]^{-1}\left(1+\epsilon Q_{c}(z)\right)^{-1} Q(z) \\
& K(\epsilon, \zeta):=A P_{0} B_{1}^{*}-\zeta A P_{0} V G_{3} V P_{0} B_{1}^{*} .
\end{aligned}
$$

By the fact that $\omega$ is of positive signature, there is a basis $\psi_{j}$ of ker $H_{\omega}$ such that $\left\langle\psi_{j}, \sigma_{3} \psi_{k}\right\rangle=\delta_{j, k}$ We can pick $U_{1}$ such that we also have $\left\langle\sigma_{3} U_{1} \psi_{j}, \psi_{k}\right\rangle=\delta_{j, k} d_{j}$, with $d_{j} \neq d_{k}$ for $j \neq k$ and $d_{j}<0$ for all $j$. Since $K(\epsilon, \zeta)$ is of rank $\operatorname{dim} \operatorname{ker}\left(H_{\omega}\right)$, we can consider the equation

$$
\begin{aligned}
& \operatorname{det}\left[\zeta^{2}+\epsilon\left(1+\epsilon Q_{c}(z)\right)^{-1} K(\epsilon, \zeta)\right]=\operatorname{det}\left[\zeta^{2}+\epsilon K(\epsilon, \zeta)\left(1+\epsilon Q_{c}(z)\right)^{-1}\right]= \\
& \operatorname{det}\left[\zeta^{2}+\epsilon\left[A \psi_{j} \frac{\left\langle B_{1}^{*} \cdot, \sigma_{3} \psi_{k}\right\rangle}{\left\langle\psi_{j}, \sigma_{3} \psi_{j}\right\rangle}-\zeta A \psi_{j} \frac{\left\langle V G_{3} V P_{0} B_{1}^{*} \cdot \sigma_{3} \psi_{k}\right\rangle}{\left\langle\psi_{j}, \sigma_{3} \psi_{j}\right\rangle}\right]+O\left(\epsilon^{2}\right)\right] \\
& =\operatorname{det}\left[\zeta^{2}+\epsilon \delta_{j, k} d_{j}+\epsilon \zeta \frac{\left\langle V G_{3} V P_{0} U_{1} \psi_{j}, \sigma_{3} \psi_{k}\right\rangle}{\left\langle\psi_{j}, \sigma_{3} \psi_{j}\right\rangle}+O\left(\epsilon^{2}\right)\right]=0 .
\end{aligned}
$$

We consider for values $\epsilon>0$ the $2 \operatorname{dim} \mathcal{V}$ solutions $\pm \sqrt{\epsilon\left|d_{j}\right|}+O\left(\epsilon^{\frac{3}{4}}\right)$. The solutions $\zeta_{j}(\epsilon)=\sqrt{\epsilon\left|d_{j}\right|}+O\left(\epsilon^{\frac{3}{4}}\right)$ with $\Re \zeta_{j}(\epsilon)>0$ yield a number of $\operatorname{dim} \mathcal{V}$ of distinct eigenvalues of $H_{\omega, \epsilon}$ given by $z_{j}(\epsilon)=\omega-\zeta_{j}^{2}(\epsilon)$ and with $\operatorname{dim} \operatorname{ker}\left(H_{\omega, \epsilon}-z_{j}(\epsilon)\right)=1$. The roots $\zeta$ of (5.4) with $\Re \zeta<0$ give singularities of the analytic continuation of $Q_{1, \epsilon}\left(\omega-\zeta^{2}\right)$ which do not correspond to eigenvalues of $H_{\omega, \epsilon}$. By the symmetry of $\sigma\left(H_{\omega, \epsilon}\right)$ with respect to the coordinate axes, all the $z_{j}(\epsilon)$ are on $\mathbb{R}$. We claim now that it is possible to choose generators $\psi_{j}(\epsilon) \in \operatorname{ker}\left(H_{\omega, \epsilon}-z_{j}(\epsilon)\right)$ such that

$$
\left\langle u, \sigma_{3} \psi_{j}(\epsilon)\right\rangle=o(1) \text { for } \epsilon \searrow 0 \text {. }
$$

If (5.5) is true, then (2.4) is true by an argument similar to the one in $\S 2$. So now we focus on (5.5). We consider a nonzero solution

$$
\left(1+A R_{0}\left(\zeta_{1}(\epsilon)\right)\left(B^{*}+\epsilon B_{1}^{*}\right)\right) \Psi_{j}(\epsilon)=0 .
$$


Then $\psi_{j}(\epsilon)=R_{0}\left(\zeta_{1}(\epsilon)\right)\left(B^{*}+\epsilon B_{1}^{*}\right) \Psi_{j}(\epsilon)$ is a nonzero element in $\operatorname{ker}\left(H_{\omega, \epsilon}-z_{j}(\epsilon)\right)$. We consider equation (5.5) using the splitting (5.2). Notice that $G_{1} V \psi=0$ for any $\psi \in \operatorname{ker} H_{\omega}$. As a consequence

$$
\begin{aligned}
& Q\left(1+A R_{0}\left(\zeta_{1}(\epsilon)\right) B^{*}\right) P= \\
& Q A\left(R_{0}\left(\zeta_{1}(\epsilon)\right)-R_{0}(0)\right) B^{*} P=-\zeta_{1} Q A G_{1} B^{*} Q+O(\epsilon)=O(\epsilon) .
\end{aligned}
$$

Similarly $P\left(1+A R_{0}\left(\zeta_{1}(\epsilon)\right) B^{*}\right) Q=O(\epsilon)$. Set $B^{*}(\epsilon)=B^{*}+\epsilon B_{1}^{*}$. Then from

$$
\left[\begin{array}{cc}
P\left(1+A R_{0}\left(\zeta_{1}(\epsilon)\right) B^{*}(\epsilon)\right) P & O(\epsilon) \\
O(\epsilon) & Q\left(1+A R_{0}\left(\zeta_{1}(\epsilon)\right) B^{*}(\epsilon)\right) Q
\end{array}\right]\left[\begin{array}{c}
P \Psi(\epsilon) \\
Q \Psi(\epsilon)
\end{array}\right]=0
$$

we get

$$
Q \Psi(\epsilon)=\left[Q\left(1+A R_{0}(0) B^{*}+o(1)\right) Q\right]^{-1} O(\epsilon) P \Psi(\epsilon)
$$

and so $\|Q \Psi(\epsilon)\|_{2} \leq C \epsilon\|P \Psi(\epsilon)\|_{2}$. Normalizing $\|P \Psi(\epsilon)\|_{2}=1$ we see that

$$
\left\langle u, \sigma_{3} \psi_{j}(\epsilon)\right\rangle=\left\langle u, \sigma_{3} R_{0}\left(\zeta_{1}(\epsilon)\right) B^{*}(\epsilon) P \Psi_{j}(\epsilon)\right\rangle+\left\langle u, \sigma_{3} R_{0}\left(\zeta_{1}(\epsilon)\right) B^{*}(\epsilon) Q \Psi_{j}(\epsilon)\right\rangle .
$$

We have $\left|\left\langle u, \sigma_{3} R_{0}\left(\zeta_{1}(\epsilon)\right) B^{*}(\epsilon) Q \Psi_{j}(\epsilon)\right\rangle\right| \leq C\|u\|_{L^{2, s}}\|Q \Psi(\epsilon)\|_{2}=O(\epsilon)$, where we use $|u(x)| \lesssim e^{-\sqrt{\omega}|x|}$ and $\langle x\rangle^{N}\left|B^{*}(\epsilon)(x)\right|<C_{N}$ for all $x \mathbb{R}^{3}$ and $\epsilon$ small. Similarly

$$
\left\langle u, \sigma_{3} R_{0}\left(\zeta_{1}(\epsilon)\right) B^{*}(\epsilon) P \Psi_{j}(\epsilon)\right\rangle=\left\langle u, \sigma_{3} R_{0}(0) B^{*} P \Psi_{j}(\epsilon)\right\rangle+o(1)=o(1)
$$

by $\sigma_{3} R_{0}(0) B^{*} P \Psi_{j}(\epsilon) \in N_{g}\left(H_{\omega}^{*}\right)$ and $u \in N_{g}^{\perp}\left(H_{\omega}^{*}\right)$.

\section{$\S 5.2 \omega$ EXCEPTIONAL OF THIRD TYPE}

We assume here that $\omega$ is an eigenvalue and a resonance. In particular we pick an appropriately normalized $\psi$ resonant vector, see Lemma $3.1[\mathrm{CP}]$. Then for $P_{0}$ and $G_{3}$ as in $\S 5.1$ we have

$$
R_{H_{\omega}}\left(\omega-\zeta^{2}\right)=\zeta^{-2} P_{0}-\zeta^{-1} P_{0} V G_{3} V P_{0}+\zeta^{-1} \psi\left\langle\quad, \sigma_{3} \psi\right\rangle+O(1)
$$

with $G_{3}(x, y)=\frac{1}{24 \pi} \operatorname{diag}(1,0)|x-y|^{2}$, i.e. the diagonal $2 \times 2$ matrix with $(1,0)$ on the diagonal. We can write

$$
Q\left(\omega-\zeta^{2}\right)=\zeta^{-2} A P_{0} B_{1}^{*}-\zeta^{-1} A P_{0} V G_{3} V P_{0} B_{1}^{*}+\zeta^{-1} A \psi\left\langle B^{*} \quad, \sigma_{3} \psi\right\rangle+Q_{c}\left(\omega-\zeta^{2}\right),
$$

where $Q_{c}\left(\omega-\zeta^{2}\right)$ admits an analytic extension for $\zeta$ around 0 . We write

$$
\begin{aligned}
& Q_{1, \epsilon}(z)=\left[1+\epsilon\left(1+\epsilon Q_{c}(z)\right)^{-1} \zeta^{-2} K(\epsilon, \zeta)\right]^{-1}\left(1+\epsilon Q_{c}(z)\right)^{-1} Q(z) \\
& K(\epsilon, \zeta):=A P_{0} B_{1}^{*}-\zeta A P_{0} V G_{3} V P_{0} B_{1}^{*}+\zeta A \psi\left\langle B_{1}^{*} \quad, \sigma_{3} \psi\right\rangle .
\end{aligned}
$$


We consider a basis $\psi_{j}$ of ker $H_{\omega}$ such that $\left\langle\psi_{j}, \sigma_{3} \psi_{k}\right\rangle=\delta_{j, k}$ and $\left\langle\sigma_{3} U_{1} \psi_{j}, \psi_{k}\right\rangle=$ $\delta_{j, k} d_{j}$, with $d_{j} \neq d_{k}$ for $j \neq k$ and $d_{j}<0$ for all $j$. We can also add that $\left\langle\sigma_{3} U_{1} \psi_{j}, \psi\right\rangle=0$ for all $j$ and $\left\langle\sigma_{3} U_{1} \psi, \psi\right\rangle=d>0$ (it is easy to see that there is a $U_{1}$ satisfying all the above hypotheses). Since $K(\epsilon, \zeta)$ is of $\operatorname{rank} 1+\operatorname{dim} \operatorname{ker}\left(H_{\omega}\right)$ we consider the equation

$$
\operatorname{det}\left[1+\epsilon \zeta^{-2} K(\epsilon, \zeta)\left(1+\epsilon Q_{c}(z)\right)^{-1}\right]=0 .
$$

This means we are considering the determinant of a matrix of the form $O\left(\epsilon^{2}\right)+$

$$
\left[\begin{array}{ccc}
\zeta^{2}+\epsilon\left(\frac{\left\langle U_{1} \psi_{j}, \sigma_{3} \psi_{k}\right\rangle}{\left\langle\psi_{j}, \sigma_{3} \psi_{j}\right\rangle}-\zeta \frac{\left\langle V G_{3} V P_{0} U_{1} \psi_{j}, \sigma_{3} \psi_{k}\right\rangle}{\left\langle\psi_{j}, \sigma_{3} \psi_{j}\right\rangle}\right) & -\zeta \epsilon \frac{\left\langle V G_{3} V P_{0} U_{1} \psi, \sigma_{3} \psi_{k}\right\rangle}{\left\langle\psi_{j}, \sigma_{3} \psi_{j}\right\rangle} \\
0 & \zeta+\epsilon\left\langle U_{1} \psi, \sigma_{3} \psi\right\rangle
\end{array}\right]
$$

that is

$$
\operatorname{det}\left(\left[\begin{array}{cc}
\zeta^{2} \delta_{j, k}+\epsilon \delta_{j, k} d_{j} & 0 \\
0 & \zeta+\epsilon d
\end{array}\right]+O\left(\epsilon \zeta+\epsilon^{2}\right)\right)=0
$$

Since $d>0$, once again there are only $\operatorname{dim} \operatorname{ker}\left(H_{\omega}\right) \operatorname{roots} \zeta_{j}(\epsilon)=\sqrt{\epsilon\left|d_{j}\right|}+O\left(\epsilon^{\frac{3}{4}}\right)$ with $\Re \zeta_{j}(\epsilon)>0$ which yield $\operatorname{dim} \operatorname{ker}\left(H_{\omega}\right)$ distinct eigenvalues of $H_{\omega, \epsilon}$ given by $z_{j}(\epsilon)=\omega-\zeta_{j}^{2}(\epsilon)$. We have $\operatorname{dim} \operatorname{ker}\left(H_{\omega, \epsilon}-z_{j}(\epsilon)\right)=1$ and $z_{j}(\epsilon) \in \mathbb{R}$ and $z_{j}(\epsilon)<\omega$. By the same argument of $\S 5.1$ it is possible to choose generators $\psi_{j}(\epsilon) \in \operatorname{ker}\left(H_{\omega, \epsilon}-z_{j}(\epsilon)\right)$ such that (5.5) holds. Then (2.4) is true by an argument similar to the one in $\S 2$.

Thus the proof of $(2.4)$ is completed

\section{REFERENCES}

[AHS] S.Agmon, I.Herbst, E.Skibsted, Perturbation of embedded eigenvalues in the generalized N-body problem, Comm. Math. Phys. 122 (1989), 411-438.

[BP] V.Buslaev, G.Perelman, On the stability of solitary waves for nonlinear Schrödinger equations, Nonlinear evolution equations (N.N. Uraltseva, eds.), Transl. Ser. 2, 164, Amer. Math. Soc., Providence, RI, 1995, pp. 75-98.

[BS] V.S.Buslaev, C.Sulem, On the asymptotic stability of solitary waves of Nonlinear Schrödinger equations, Ann. Inst. H. Poincaré. An. Nonlin. 20 (2003), 419-475.

[CL] T.Cazenave, P.L.Lions, Orbital stability of standing waves for nonlinear Schrödinger equations, Comm. Math. Phys. 85 (1982), 549-561.

[CHM] C.Cruz-Sampedro, I.Herbst, R.Martinez-Avendano, Perturbations of the Wigner-Von Neuman potential leaving the embedded eigenvalue fixed, Ann H. Poincare 3 (2002), 331-346.

[CoP $] \quad$ A.Comech, D.Pelinovsky, Purely nonlinear instability of standing waves with minimal energy, Comm. Pure Appl. Math. 56 (2003), 1565-1607.

[Cu1] S.Cuccagna, On asymptotic stability in energy space of ground states of NLS in $1 D$, http://arxiv.org/.

[Cu2] Stabilization of solutions to nonlinear Schrödinger equations, Comm. Pure App. Math. 54 (2001), 1110-1145. 
[Cu3] - On asymptotic stability of ground states of NLS, Rev. Math. Phys. 15 (2003), 877-903.

[CM] S.Cuccagna, T.Mizumachi, On asymptotic stability in energy space of ground states for nonlinear Schrödinger equations, http://arxiv.org/.

[CP] S.Cuccagna, D.Pelinovsky, Bifurcations from the endpoints of the essential spectrum in the linearized nonlinear Schrödinger problem, J. Math. Phys. 46 (2005), 053520.

[CPV] S.Cuccagna, D.Pelinovsky, V.Vougalter, Spectra of positive and negative energies in the linearization of the NLS problem, Comm. Pure Appl. Math. 58 (2005), 1-29.

[CT] S.Cuccagna, M.Tarulli, On asymptotic stability in energy space of ground states of NLS in 2D, http://arxiv.org/.

[JK] A.Jensen, T.Kato, Spectral properties of Schrödinger operators and time decay of the wave functions, Duke Math. J. 46 (1979), 583-611.

[J] C.K.R.T.Jones, An instability mechanism for radially symmetric standing waves of nonlinear Schrödinger equations, Jour. Diff. Eq. 71 (1988), 34-62.

[Gz] Zhou Gang, Perturbation Expansion and N-th Order Fermi Golden Rule of the Nonlinear Schrödinger Equations, J. Math. Phys. 48 (2007), 053509.

[GS] Zhou Gang, I.M.Sigal, Relaxation of Solitons in Nonlinear Schrödinger Equations with Potential, http://arxiv.org/abs/math-ph/0603060.

[Gr1] M.Grillakis, Analysis of the linearization around a critical point of an infinite dimensional Hamiltonian system, Comm. Pure Appl. Math 43 (1990), 299-333.

[Gr2] Linearized instability for nonlinear Schrödinger and Klein Gordon equations, Comm. Pure Appl. Math. 41 (1988), 747-774.

[GSS1] M.Grillakis, J.Shatah, W.Strauss, Stability of solitary waves in the presence of symmetries, I, Jour. Funct. An. 74 (1987), 160-197.

[GSS2] Stability of solitary waves in the presence of symmetries, II, Jour. Funct. An. 94 (1990), 308-348.

[M1] T.Mizumachi, A remark on linearly unstable standing wave solutions to NLS, Nonlinear Analysis 64 (2006), 657-676.

[M2] _ Vortex solitons for 2D focusing Nonlinear Schrödinger Equations, Diff. Integral Equations 18 (2005), 431-450.

[M3] _ Instability of bound states for 2D Nonlinear Schrödinger Equations, Discr. Cont. Dyn. Systems 13 (2005), 413-428.

[M4] Instability of vortex solitons for $2 D$ focusing $N L S$.

[Si] I.M.Sigal, Nonlinear wave and Schrödinger equations. I. Instability of periodic and quasi- periodic solutions, Comm. Math. Phys. 153 (1993), 297-320.

[SW1] A.Soffer, M.Weinstein, Selection of the ground state for nonlinear Schrödinger equations, Rev. Math. Phys. 16 (2004), 977-1071.

[SW2] - Resonances, radiation damping and instability in Hamiltonian nonlinear wave equations, Invent. Math. 136 (1999), 9-74.

[T] T.P.Tsai, Asymptotic dynamics of nonlinear Schrödinger equations with many bound states, J. Diff. Eq. 192 (2003), 225-282.

[TY1] T.P.Tsai, H.T.Yau, Asymptotic dynamics of nonlinear Schrödinger equations: resonance dominated and radiation dominated solutions, Comm. Pure Appl. Math. 55 (2002), 153-216.

[TY2] , Relaxation of excited states in nonlinear Schrödinger equations, Int. Math. Res. Not. 31 (2002), 1629-1673.

[TY3] Classification of asymptotic profiles for nonlinear Schrödinger equations with small initial data, Adv. Theor. Math. Phys. 6 (2002), 107-139.

[TY4] Stable directions for excited states of nonlinear Schrödinger equations, Comm. Partial Diff. Eq. 27 (2002), 2363-2402. 
[We1] M.Weinstein, Lyapunov stability of ground states of nonlinear dispersive equations, Comm. Pure Appl. Math. 39 (1986), 51-68.

[We2] Modulation stability of ground states of nonlinear Schrödinger equations, Siam J. Math. Anal. 16 (1985), 472-491.

Dismi University of Modena and Reggio Emilia, via Amendola 2, Padiglione Morselli, RegGio Emilia 42100 Italy

E-mail address: cuccagna.scipio@unimore.it 